This item was submitted to Loughborough's Research Repository by the author.

Items in Figshare are protected by copyright, with all rights reserved, unless otherwise indicated.

Transcritical flow of a stratified fluid over topography: analysis of the forced Gardner equation

PLEASE CITE THE PUBLISHED VERSION

http://dx.doi.org/10.1017/jfm.2013.556

PUBLISHER

(c) Cambridge University Press

VERSION

AM (Accepted Manuscript)

LICENCE

CC BY-NC-ND 4.0

REPOSITORY RECORD

Kamchatnov, A.M., Y.-H. Kuo, Tai-Chia Lin, T.-L. Horng, S.-C. Gou, Richard Clift, G.A. El, and Roger H.J. Grimshaw. 2019. "Transcritical Flow of a Stratified Fluid over Topography: Analysis of the Forced Gardner Equation”. figshare. https://hdl.handle.net/2134/14279. 


\title{
Transcritical flow of a stratified fluid over topography: analysis of the forced Gardner equation
}

\author{
A. M. KAMCHATNOV ${ }^{1}$, Y.-H. KUO ${ }^{2}$, T.-C. LIN $^{3}$, T.-L. HORNG ${ }^{4}$, \\ S.-C. GOU ${ }^{5}$, R. CLIFT ${ }^{6}$, G. A. EL ${ }^{6}$, R. H. J. GRIMSHAW \\ ${ }^{1}$ Institute of Spectroscopy, Russian Academy of Sciences, Troitsk, Moscow, 142190, Russia \\ ${ }^{2}$ Department of Mathematics, National Taiwan University, Taipei, Taiwan, \\ ${ }^{3}$ Institute of Applied Mathematical Sciences, National Taiwan University, Taipei, Taiwan \\ ${ }^{4}$ Department of Applied Mathematics, Feng Chia University, Taichung 40724, Taiwan \\ ${ }^{5}$ Department of Physics, National Changhua University of Education, \\ Changhua 50058, Taiwan \\ ${ }^{6}$ Department of Mathematical Sciences, Loughborough University, \\ Loughborough LE11 3TU, UK
}

(Received )

Transcritical flow of a stratified fluid past a broad localised topographic obstacle is studied analytically in the framework of the forced extended Korteweg-de Vries (eKdV), or Gardner, equation. We consider both possible signs for the cubic nonlinear term in the Gardner equation corresponding to different fluid density stratification profiles. We identify the range of the input parameters: the oncoming flow speed (the Froude number) and the topographic amplitude, for which the obstacle supports a stationary localised hydraulic transition from the subcritical flow upstream to the supercritical flow downstream. Such a localised transcritical flow is resolved back into the equilibrium flow state away from the obstacle with the aid of unsteady coherent nonlinear wave structures propagating upstream and downstream. Along with the regular, cnoidal undular bores occurring in the analogous problem for the single-layer flow modeled by the forced $\mathrm{KdV}$ equation, the transcritical internal wave flows support a diverse family of upstream and downstream wave structures, including kinks, rarefaction waves, classical undular bores, reversed and trigonometric undular bores, which we describe using the recent development of the nonlinear modulation theory for the (unforced) Gardner equation. The predictions of the developed analytic construction are confirmed by direct numerical simulations of the forced Gardner equation for a broad range of input parameters.

\section{Introduction}

The problem of weakly nonlinear long wave evolution over localized obstacles is of fundamental importance in various branches of fluid mechanics and has a number of applications in oceanography and meteorology. The particular case of one-dimensional flow propagation over a broad ridge is quite remarkable due to the existence of a generic mechanism of the wave generation when the speed of the flow becomes close to the local speed of linear long waves in the reference frame of the obstacle. This mechanism is essentially nonlinear (see, e.g., Akylas (1984); Cole (1985)) and its mathematical description requires inclusion of appropriately balanced forcing, nonlinear and dispersive terms in the corresponding asymptotic model.

The prominent feature of a near-critical flow past broad obstacles is the formation of a smooth steady (hydraulic) transition over the obstacle region so that the flow in front of 
the obstacle is subcritical and after the obstacle supercritical (see Baines (1995) and references therein). The upstream and downstream non-equilibrium states are resolved back into the equilibrium flow away from the obstacle with the aid of unsteady nonlinear wave trains, or undular bores. The general mathematical framework for the study of transcritical weakly nonlinear and weakly dispersive fluid flows was introduced in Grimshaw \& Smyth (1986). In this work a combination of the dispersionless hydraulic solution of the forced Korteweg-de Vries (KdV) equation for the near-field and the solutions of the KdVWhitham equations describing the modulations in the undular bores propagating away from the obstacle were used. This Grimshaw-Smyth (GS) formulation has been widely used in further studies of transcritical shallow-water flows (see Madsen \& Hansen (2012) and references therein) but has also proved to be relevant to other physical contexts such as superfluids, Leszczyszyn et al. (2009), and nonlinear optics, Wan et al. (2010), when the "fluid" dynamics is governed by the defocusing Gross-Pitaevskii/nonlinear Schrödinger equation.

If the forcing amplitude is small, the dynamics of the transcritical shallow-water flow (both for surface and internal waves) is typically governed by the forced KdV equation (see the reviews Baines (1995); Grimshaw (2001); Helfrich \& Melville (2006); Apel et al. (2007)). However, for sufficiently large forcing or for special conditions of the background density stratification the description of internal waves generated in stratified fluid flows often requires the inclusion of higher-order nonlinear terms resulting, for near-critical flows, in the forced extended $\mathrm{KdV}(\mathrm{eKdV})$, or Gardner, equation, which in non-dimensional variables has the form (see, e.g., Kakutani \& Yamasaki (1978); Melville \& Helfrich (1987))

$$
-u_{t}-\Delta u_{x}+6 u u_{x}-6 \alpha u^{2} u_{x}+u_{x x x}+G_{x}=0 .
$$

Here $u$ denotes an appropriate field variable (e.g., the interfacial displacement in a twolayer fluid), $\alpha$ measures the relative value of the cubic nonlinear term vis-a-vis the quadratic nonlinear term, $\Delta$ is the deviation of the incident flow velocity from the long wave phase speed and $G(x)$ represents the localized topographic forcing, that is we assume that

$$
G(x) \rightarrow 0 \quad \text { at } \quad x \rightarrow \pm \infty
$$

$G(x)$ can be considered as vanishing outside the region $|x| \gtrsim l$ and having its maximal value $G_{m}$ at $x=0$. We shall be interested here only in the positive forcing case, $G(x)>0$. In numerical simulations $G(x)$ is often taken as

$$
G(x)=G_{m} \exp \left(-x^{2} / l^{2}\right) .
$$

At the initial moment of time it is assumed that

$$
u(x, 0)=0,
$$

which corresponds to "turning on" the forcing at $t=0$. Thus the flow satisfies the boundary condition

$$
u(x, t) \rightarrow 0 \text { for } \quad|x| \rightarrow \infty .
$$

The problem (1.1), (1.2), (1.4), (1.5) was considered using a number of approaches, but mostly numerically, in Melville \& Helfrich (1987), Marchant \& Smyth (1990) and Grimshaw et al. (2002). In Marchant \& Smyth (1990) an analytical treatment of the forced Gardner equation was restricted to the case of small positive values of $\alpha$ in (1.1) which enables one to capture only the small quantitative corrections to the wave regimes described by the forced KdV equation. In Grimshaw et al. (2002) the transcritical hydraulic solution of the forced Gardner equation was constructed for finite values of $\alpha$ 
but the unsteady wavetrains generated upstream and downstream were treated only numerically. This latter study revealed a broad variety of the wave regimes realised in transcritical internal wave flows, part of which were observed in an earlier work Melville \& Helfrich (1987). Remarkably, some of these numerically obtained regimes for the forced Gardner equation were sharply different from those occurring in the forced KdV dynamics Grimshaw \& Smyth (1986). In particular, the numerical solutions of the forced Gardner equation (1.1) with $\alpha>0$ presented in Melville \& Helfrich (1987) and Grimshaw et al. (2002) showed that, for a certain range of the problem input parameters $G_{m}$ and $\Delta$ the upstream structure consists of the combination of a rarefaction wave and the monotone bore solution of the Gardner equation, rather than the more usual cnoidal undular bore observed in the counterpart KdV setting, when $\alpha=0$. Other generated patterns occurring in the numerical simulations in Grimshaw et al. (2002) include solitary wavetrains and irregularly generated waves of variable amplitude which may continually interact. Unlike the forced $\mathrm{KdV}$ equation, the forced Gardner equation was shown to support propagating wave structures in subcritical and supercritical flow regimes. We stress that the need for using the Gardner equation instead of the simpler KdV equation for the description of internal waves is supported by many observational and experimental data (see e.g. Michallet \& Barthélemy (1998), Holloway et al. (2001), Helfrich \& Melville (2006) and references therein).

In this paper we restrict our study to the transcritical flow regimes when the obstacle supports a stationary localised hydraulic transition from the subcritical flow upstream to the supercritical flow downstream. In this case an analytical description of the unsteady waves generated upstream and downstream in the framework of the general GS setting becomes possible in principle. The practical realisation of this possibility, however, had been hindered until recently due to the unavailability of the full modulation (Whitham) theory for the Gardner equation.

The modulation theory for the Gardner equation was developed in the recent study Kamchatnov et al. (2012) where the complete classification of the initial step resolution (Riemann) problem was constructed. The generated structures, along with the KdV type cnoidal undular bores, include nonlinear "trigonometric" bores, kinks or solibores, rarefaction waves and composite solutions representing various combinations of the above structures. Note that the term "solibore" has been used in the literature with several different meanings, but here we use it to mean only the kink solution of the Gardner equation described originally by Kakutani \& Yamasaki (1978); that is, the monotone bore solution of the Gardner equation. In the current paper, we utilise the analytical results of Kamchatnov et al. (2012) to describe and classify the wave patterns occurring in transcritical internal wave flows over broad localised topography. We consider both signs of the coefficient of the cubic nonlinear term, $\alpha$ in (1.1) corresponding to different background density stratification conditions (see e.g. Grimshaw (2001)).

The structure of the paper is as follows. In section 2 we present a detailed study of the solutions to the forced Gardner equation (1.1) in the hydraulic (steady dispersionless) approximation. These are needed, in particular, to derive the values of the upstream and downstream hydraulic jumps forming in the transcritical flow regime. In section 3 the full classification of solutions to the initial step resolution (Riemann) problem for the Gardner equation is presented following the recent study by Kamchatnov et al. (2012). The results of sections 2 and 3 are then used in sections 4 and 5 to describe the unsteady wave patterns occurring in the dispersive resolution of the upstream and downstream hydraulic jumps in the transcritical flows governed by the Gardner equation (1.1) with a broad forcing for both signs of $\alpha$. The predictions of the analytical theory developed in 
sections 3-5 are confirmed by the direct numerical simulations of the problem (1.1)-(1.5). In section 6 we present conclusions.

\section{Hydraulic approximation}

In this section we present an analysis of the solutions to the forced Gardner equation in the hydraulic approximation which is the key to the GS construction employed in this paper. The conditions for the existence of the steady localised transcritical hydraulic solution giving rise to the generation of unsteady wave structures upstream and downstream of the obstacle were identified in Grimshaw et al. (2002). Below we review, detail and extend the results of Grimshaw et al. (2002) by identifying, within the region of existence of the transcritical hydraulic transition, the further three subregions (for either sign of $\alpha$ ) corresponding to various unsteady wave regimes occurring away from the obstacle. This is done using the full classification of the solutions to the Riemann step problem for the Gardner equation obtained in Kamchatnov et al. (2012).

\subsection{Subcritical and supercritical flows}

We assume that in non-dimensional units $l \gg 1$. It is then natural to expect that the topographic forcing results in a disturbance $u$ also with the characteristic length $\sim l$. Then the dispersion term $u_{x x x} \sim l^{-3}$ is relatively small compared with the other terms $\sim l^{-1}$ and we can describe the motion by the dispersionless limit of (1.1)

$$
-u_{t}-\Delta u_{x}+6 u u_{x}-6 \alpha u^{2} u_{x}+G_{x}=0 .
$$

The "switching on" the forcing generates the disturbance in the region $|x| \lesssim l$ of the location of the topography. One can then expect that some part of the disturbance will leave this region with the group velocity and, in the limit $t \rightarrow \infty$, can be neglected whereas the remaining part can be described by a stationary solution of equation (2.1), that is

$$
-\Delta u_{x}+6 u u_{x}-6 \alpha u^{2} u_{x}+G_{x}=0,
$$

with the boundary condition (1.5). Approximation (2.2) is usually called the hydraulic approximation. Integrating (2.2) and using the boundary condition (1.5),

$$
-\Delta u+3 u^{2}-2 \alpha u^{3}+G(x)=0,
$$

yields the dependence $u(x)$ in an implicit form for a given function $G(x)$. Let us consider the conditions for the existence of such a solution for all $-\infty<x<\infty$.

Equation (2.3) can be written in the form,

$$
F(u)=\Delta u-3 u^{2}+2 \alpha u^{3}=G(x),
$$

where the polynomial $F(u)$ has three roots, one of which is $u=0$, corresponding to the equilibrium state at $x \rightarrow \pm \infty$. If the other two roots are real, then, since $G>0$, the solution of (2.3) is possible for $F(u)>0$ only, and it can be found for all $x \in(-\infty, \infty)$ provided the following condition is satisfied:

$$
G_{m} \leq F_{m},
$$

where $G_{m}$ is the amplitude of the forcing and $F_{m}$ is a local maximum of the function $F(u)$, which exists for

$$
\Delta<\frac{3}{2 \alpha} \text { for } \alpha>0, \quad \text { or } \quad \Delta>\frac{3}{2 \alpha} \quad \text { for } \quad \alpha<0 .
$$




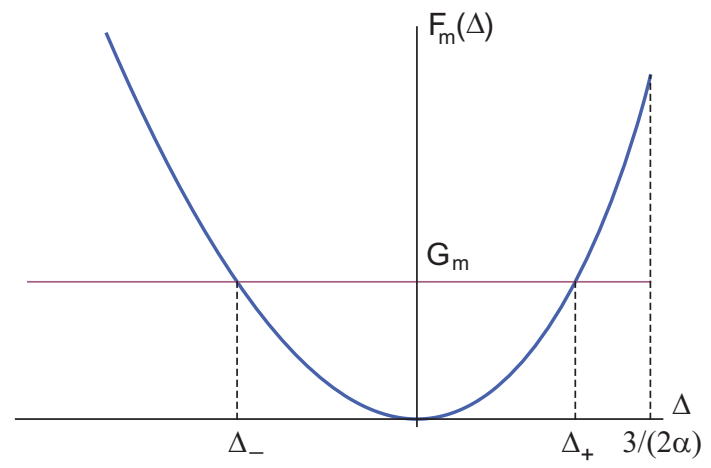

Figure 1. Plot of the function $F_{m}(\Delta)$ defined by Eq. (2.8) with $\alpha>0$. Subcritical $\Delta<\Delta_{-}$and supercritical $\Delta>\Delta_{+}$regions correspond to the values of $\Delta$ when the condition $G_{m} \leq F_{m}(\Delta)$ is satisfied.

Otherwise this maximum disappears and the hydraulic solution is guaranteed to exist for any value of $G_{m}$.

It is easy to find that $F(u)$ reaches its maximum value at

$$
u_{m}=\frac{1}{2 \alpha}\left(1-\sqrt{1-\frac{2 \alpha \Delta}{3}}\right)
$$

and it is given by the formula

$$
F_{m}(\Delta)=F\left(u_{m}\right)=-\frac{1}{2 \alpha^{2}}\left[1-\left(1-\frac{2 \alpha \Delta}{3}\right)^{3 / 2}\right]+\frac{\Delta}{2 \alpha} .
$$

For future reference we indicate here the value

$$
u_{l}=\frac{1}{2 \alpha}\left[1+\sqrt{1-\frac{2 \alpha \Delta}{3}}\right]
$$

at which $F(u)$ has a local minimum equal to

$$
F_{l}(\Delta)=F\left(u_{l}\right)=-\frac{1}{2 \alpha^{2}}\left[1+\left(1-\frac{2 \alpha \Delta}{3}\right)^{3 / 2}\right]+\frac{\Delta}{2 \alpha} .
$$

The dependence of $F_{m}(\Delta)$ is illustrated in Fig. 1 for the case $\alpha>0$. The condition (2.5) is fulfilled in the regions

$$
-\infty<\Delta<\Delta_{-} \quad \text { and } \quad \Delta_{+}<\Delta<\frac{3}{2 \alpha}
$$

where $\Delta_{ \pm}$are determined by the equation $F_{m}(\Delta)=G_{m}$, that is,

$$
-\frac{1}{2 \alpha^{2}}\left[1-\left(1-\frac{2 \alpha \Delta}{3}\right)^{3 / 2}\right]+\frac{\Delta}{2 \alpha}=G_{m} .
$$

When $\alpha>0$ then $\Delta_{+} \leq 3 / 2 \alpha$ for $G_{m} \leq 1 / 4 \alpha^{2}$ and increases monotonically as $G_{m}$ increases, and $\Delta_{+}=3 / 2 \alpha$ for $G_{m}>1 / 4 \alpha^{2}$, while when $\alpha<0, \Delta_{+}$increases monotonically as $G_{m}$ increases. Similarly, when $\alpha>0$ then $\Delta_{-}$decreases monotonically as $G_{m}$ increases, while when $\alpha<0, \Delta_{-} \geq 3 / 2 \alpha$ for $G_{m} \leq 1 / 4 \alpha^{2}$ and decreases monotonically as $G_{m}$ increases, and $\Delta_{-}=3 / 2 \alpha$ for $G_{m}>1 / 4 \alpha^{2}$. The region $-\infty<\Delta<\Delta_{-}$is subcritical and the region $\Delta_{+}<\Delta<+\infty$ is supercritical. In both these regions a stationary 

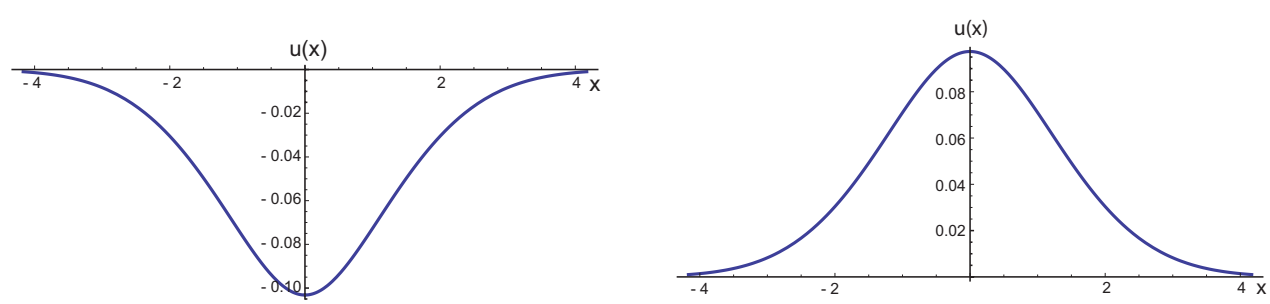

Figure 2. The hydraulic solution $u(x)$ for subcritical and supercritical values of $\Delta: \Delta=-1.3$ (left) and $\Delta=1.3$ (right). For $G_{m}=0.1$ and $\alpha=1$ the critical values are equal to $\Delta_{-}=-1.1555$ and $\Delta_{+}=1.0177$; the width of the forcing (1.3) equals to $l=2$.

global solution exists in the hydraulic approximation with $u$ continuously depending on $x$ on the entire axis. These solutions are illustrated in Fig. 2. As we can see, the form of the near-field reproduces qualitatively the form of the obstacle and the polarity of the disturbance depends on the value of $\Delta$. It is a localised depression for subcritical $\Delta$ and a localised elevation for supercritical $\Delta$. The supercritical (subcritical) solution has a maximum (minimum) $u_{m}$.

It readily follows from $(2.12)$ that the dependence of $\Delta_{ \pm}$on $G_{m}$ can be expressed in an explicit form,

$$
\Delta_{ \pm}=\frac{1}{\alpha} \Phi_{ \pm}\left(\alpha^{2} G_{m}\right) \quad \text { for } \quad \alpha>0 \quad \text { or } \quad \Delta_{ \pm}=\frac{1}{\alpha} \Phi_{\mp}\left(\alpha^{2} G_{m}\right) \quad \text { for } \quad \alpha<0
$$

where $\Phi_{ \pm}(y)$ are determined as functions inverse to

$$
y=\Phi^{-1}(x)=\frac{1}{2}\left[x-1+\left(1-\frac{2 x}{3}\right)^{3 / 2}\right] .
$$

They can be represented in the explicit form,

$$
\Phi_{ \pm}(y)=\frac{3}{8}\left(1-2 \sqrt{1+32 y} \cos \frac{\theta(y) \pm \pi}{3}\right),
$$

where

$$
\theta(y)=\arccos \left(\frac{1-80 y-128 y^{2}}{(1+32 y)^{3 / 2}}\right), \quad 0 \leq y \leq 1 / 4 .
$$

In the limit of small $|\alpha|$ we find the series expansion

$$
F_{m}(\alpha, \Delta) \cong \frac{\Delta^{2}}{12}+\frac{\alpha \Delta^{3}}{108}+\frac{\alpha^{2} \Delta^{4}}{432}+\ldots
$$

and, correspondingly,

$$
\begin{aligned}
& \Delta_{-} \cong-\sqrt{12 G_{m}}-\frac{2 \alpha G_{m}}{3}+\frac{4 \alpha^{2} G_{m}^{3 / 2}}{9 \sqrt{3}}+\ldots, \\
& \Delta_{+} \cong \sqrt{12 G_{m}}-\frac{2 \alpha G_{m}}{3}-\frac{4 \alpha^{2} G_{m}^{3 / 2}}{9 \sqrt{3}}+\ldots,
\end{aligned}
$$

The first term of this expansion corresponds to the forced KdV result of Grimshaw \& Smyth (1986).

\subsection{Transcritical region}

If $\Delta$ is located in the transcritical region

$$
\Delta_{-}<\Delta<\Delta_{+}
$$


then the global smooth steady hydraulic solution defined for all $x \in(-\infty,+\infty)$ and described in section 2.1, ceases to exist. Nevertheless, in the region $-l \lesssim x \lesssim l$ of the obstacle's location the hydraulic approximation can still be applicable, but now, considered globally, the hydraulic solution no longer satisfies the equilibrium boundary condition (1.5). Rather, instead there exists a region of the flow parameters when the hydraulic solution tends to some nonzero constant values,

$$
u(x) \rightarrow \begin{cases}u^{u}, & x \rightarrow-\infty \\ u^{d}, & x \rightarrow+\infty\end{cases}
$$

where the upstream and downstream values $u^{u}$ and $u^{d}$ respectively are determined by these parameters. Of course, the limits $x \rightarrow \pm \infty$ have here a formal sense; the limiting values $u^{u, d}$ are practically reached at $x \sim \pm l$ (we remind that $l \gg 1$ ) and to satisfy the equilibrium boundary conditions at infinity, upstream and downstream undular bores must be inserted (see Grimshaw \& Smyth (1986)).

Since the boundary condition (1.5) is no longer applicable in the transcritical region, one should use instead of (2.3) the general integral of Eq. (2.2),

$$
F(u)+C=G(x),
$$

where $C$ is an integration constant, and can be found in the following way. Let the maximal value $G_{m}$ of $G(x)$ be located at $x=0$, so that $G_{x}(0)=0$. We look for the transcritical solution with $u_{x}(0) \neq 0$; hence Eq. (2.2) yields

$$
u_{m} \equiv u(0)=\frac{1}{2 \alpha}\left(1-\sqrt{1-\frac{2 \alpha \Delta}{3}}\right)
$$

which coincides with (2.7). Hence, $u(x)$ is determined by the equation

$$
\begin{gathered}
F(u)-G(x)=F_{m}(\Delta)-G_{m}, \\
\text { or } 2 \alpha u^{3}-3 u^{2}+\Delta u-G(x)=-\frac{1}{2 \alpha^{2}}\left[1-\left(1-\frac{2 \alpha \Delta}{3}\right)^{3 / 2}\right]+\frac{\Delta}{2 \alpha}-G_{m} .
\end{gathered}
$$

In the formal limit $x \rightarrow \pm \infty$ (actually, for $|x| \sim l$ ) we have $G(x) \rightarrow 0$ and the limiting values $u^{u, d}$ of $u$ are the roots of the equation

$$
2 \alpha u^{3}-3 u^{2}+\Delta u=-\frac{1}{2 \alpha^{2}}\left[1-\left(1-\frac{2 \alpha \Delta}{3}\right)^{3 / 2}\right]+\frac{\Delta}{2 \alpha}-G_{m} .
$$

Although this equation has three roots, only two of them neighboring $u_{m}$ have physical meaning since they correspond to the limiting values of a continuous solution of Eq. (2.24) in the whole range of the values of $x$ in the interval $[-l, l]$. The upstream and downstream values $u^{u}$ and $u^{d}$ are ordered according to

$$
u^{d}<u_{m}<u^{u},
$$

in order to ensure that the corresponding resolution of the jumps from $u^{u, d}$ back to the undisturbed equilibrium $u=0$ is made by structures which propagate upstream and downstream respectively. Equation (2.25) supplemented by this condition determines the roots $u^{u, d}$ without any ambiguity provided $\Delta$ satisfies the condition of their reality. Indeed, typical graphs of the function of $F(u)$ for its characteristic parameters are shown in Fig. 3 for the case $\alpha>0$. Similar graphs can be readily plotted for the case $\alpha<0$.

It is clear that equation (2.25) has two real roots $u^{u}$ and $u^{d}$ if $G_{m}$ satisfies the following 

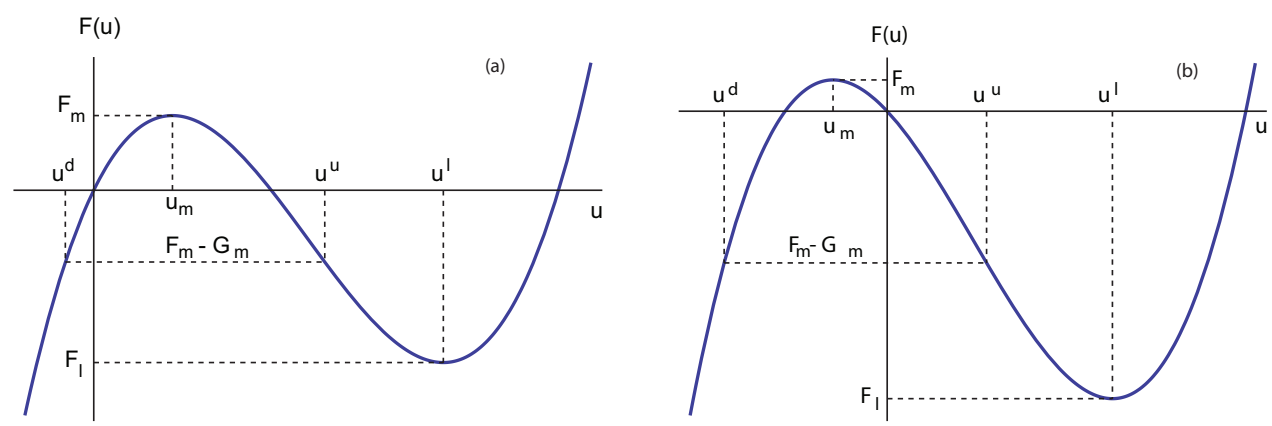

FiguRE 3. Plots of the function $F(u)$ with $\alpha>0$ for (a) $\Delta>0$; and (b) $\Delta<0$.

condition

$$
F_{m}-G_{m}>F_{l}
$$

where $F_{m}$ is the local maximal value (2.8) and $F_{l}$ is the local minimal value (2.10), which yields

$$
G_{m}<\frac{1}{\alpha^{2}}\left(1-\frac{2 \alpha \Delta}{3}\right)^{3 / 2} .
$$

It is readily shown that the transcritical regime is indeed $\Delta_{-}<\Delta<\Delta_{+}$where the limiting values $\Delta_{ \pm}\left(G_{m}\right)$ are precisely those found in section 2.1 as the lower and upper boundaries of the supercritical and subcritical regimes respectively. These boundaries are shown in the transcritical flow-regime diagrams in Figs. 4a and $4 \mathrm{~b}$ by thick lines. We note that the diagrams in Fig. 4 are universally presented in terms of $\alpha^{2} G_{m}$ and $|\alpha| \Delta$ so that they do not depend on the stratification parameter $\alpha$. We also mention that the "cut-off" of the upper boundary of the transcritical region at some $|\alpha| \Delta>0$ is consistent with the behaviour of transcritical flow-regime diagram for the fully nonlinear transcritical two-layer flows (see e.g. Baines (1984) and White \& Helfrich (2012)).

Next, from Eq. (2.28) we conclude that the transcritical region is split into two subregions by the line $\Delta_{+}^{\prime}$ for $\alpha>0$ or by the line $\Delta_{-}^{\prime}$ for $\alpha<0$ determined by the equations

$$
G_{m}=\frac{1}{\alpha^{2}}\left(1-\frac{2 \alpha \Delta}{3}\right)^{3 / 2} \quad \text { or } \quad \Delta_{\operatorname{sgn}(\alpha)}^{\prime}=\frac{3}{2 \alpha}\left[1-\left(\alpha^{2} G_{m}\right)^{2 / 3}\right] .
$$

These boundaries are shown in Fig. 4 by thick dashed lines. They appear for $\alpha^{2} G_{m}>1 / 8$ and mean that, if $\alpha>0$ then in the domain $\Delta_{-}<\Delta<\Delta_{+}^{\prime}$ both roots $u^{u, d}$ exist whereas in $\Delta_{+}^{\prime}<\Delta<\Delta_{+}$the root $u^{u}$ disappears and the localised hydraulic solution with different limiting values at $x \rightarrow \pm \infty$ ceases to exist. In a similar way, if $\alpha<0$, then both roots $u^{u, d}$ exist for $\Delta_{-}^{\prime}<\Delta<\Delta_{+}$, which corresponds to the existence of the hydraulic solution with different asymptotic values at $x \rightarrow \pm \infty$, but such a solution does not exist if $\Delta_{-}<\Delta<\Delta_{-}^{\prime}$. This is in contrast with the forced KdV equation dynamics (see Grimshaw \& Smyth (1986)), where the steady localised hydraulic solution exists within entire transcritical region (although the time of establishment of this steady solution could be very long for the regimes close to the boundaries of the transcritical region).

Typical behaviours of $u^{u}, u^{d}$ as functions of $\Delta$ at a fixed $G_{m}$ are illustrated in Fig. 5 (note that the plots shown in Fig. 5 correspond to $G_{m} \alpha^{2}<1 / 8$ so the transcritical hydraulic solution is valid in the whole range $\Delta_{-}<\Delta<\Delta_{+}$- see Fig. 4). In the case of small $G_{m}$ we have the series expansions for $u^{u, d}$,

$$
u^{u, d}=\frac{1}{6}\left[\Delta \pm \sqrt{12 G_{m}}+\frac{\alpha}{6}\left(\Delta^{2} \pm \Delta \sqrt{12 G_{m}}+4 G_{m}\right)\right]+\ldots
$$



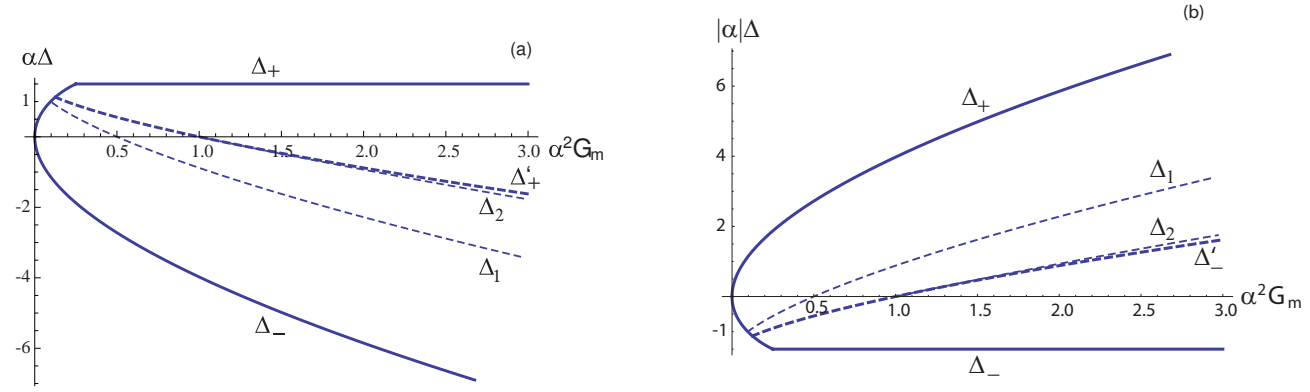

FigURE 4. Universal transcritical flow-regime diagrams in terms of $\alpha^{2} G_{m}$ and $|\alpha| \Delta$ for (a) $\alpha>0$, and (b) $\alpha<0$.
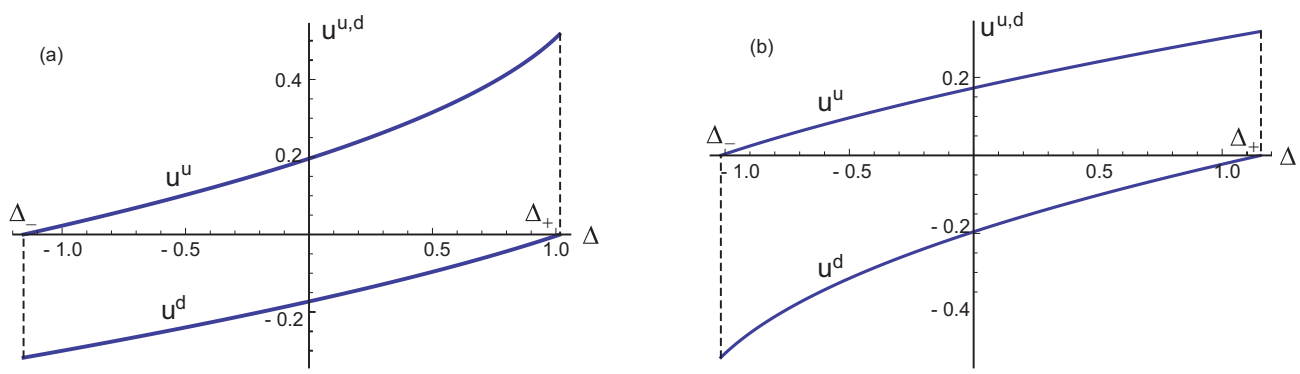

FIgURE 5. Typical plots of $u^{u}$ and $u^{d}$ as functions of $\Delta$ for (a) $\alpha=+1$; and (b) $\alpha=-1$. In both cases the forcing amplitude $G_{m}=0.1$.

where the upper sign corresponds to $u^{u}$ and the lower one to $u^{d}$. The first two terms in (2.30) agree with the KdV approximation of Grimshaw \& Smyth (1986).

When $u^{u}$ and $u^{d}$ as solutions of the equation (2.25) are known, we are in position to construct the unsteady solutions connecting these values with the equilibrium state $u=0$ at $x \rightarrow \pm \infty$ and providing thus a closure for the localised transcritical hydraulic transition. Importantly, since the corresponding unsteady structures are generated away from the topography forcing, their description is to be made in the framework of the homogeneous, unforced Gardner equation. More specifically, we shall be interested in the asymptotic solutions to the initial step problem for the Gardner equation assuming that for sufficiently large times one can neglect the width of the obstacle compared with the width of the generated expanding wave structure.

As is well known, a single-layer shallow-water flow in the transcritical regime generates upstream and downstream undular bores, which in the weakly nonlinear approximation are asymptotically described by the modulated periodic solutions of the (unforced) KdV equation; one of the bores being attached to the obstacle and realised only partially (see Grimshaw \& Smyth (1986) and Smyth (1987)). In contrast to the KdV equation, the Gardner equation supports a broad variety of unsteady solutions occurring in the step Riemann problem, which, along with the KdV type "cnoidal" bores, include solibores, rarefaction waves, nonlinear trigonometric bores and compound solutions representing combinations of the above structures. These solutions were recently constructed by the present authors in Kamchatnov et al. (2012), and below we shall take advantage of the results of that work to describe weakly nonlinear unsteady wave regimes occurring in transcritical flows.

As follows from the results of Kamchatnov et al. (2012), the type of the solution to 
the force-free Gardner equation arising in the resolution of an initial step

$$
u(x, 0)= \begin{cases}u^{-} & \text {for } \quad x<0, \\ u^{+} & \text {for } \quad x>0\end{cases}
$$

strongly depends on the positions of boundary values $u^{ \pm}$relative to the turning point $u=1 /(2 \alpha)$ of the dispersionless limit characteristic velocity $c(u)=\Delta-6 u(1-\alpha u)$ of the Gardner equation (1.1). We shall outline the Riemann problem classifications for $\alpha>0$ and $\alpha<0$ in the next section; here we only take advantage of some basic results from Kamchatnov et al. (2012) enabling one to identify the subdomains within the transcritical regions $\Delta_{-}<\Delta<\Delta_{+}^{\prime}(\alpha>0)$ and $\Delta_{-}^{\prime}<\Delta<\Delta_{+}(\alpha<0)$ corresponding to qualitatively different behaviours of the unsteady solutions away from the obstacle.

If $\alpha>0$ then downstream of the obstacle one always has $u^{-}=u^{d}<0, u^{+}=0$, which corresponds to the cnoidal undular bore of 'normal' polarity (i.e. having a 'bright' solitary wave of elevation at the leading edge) propagating to the right (note that the Gardner equation (1.1) with zero forcing term is related to the Gardner equation in the standard form used in Kamchatnov et al. (2012) by a simple linear transformation of the independent variables, see the next section). However, upstream of the obstacle several different regimes are possible depending on the value of $u^{u}$. These regimes are separated by the lines in the $\left(\alpha^{2} G_{m}, \alpha \Delta\right)$-plane corresponding to $u^{u}=1 /(2 \alpha)$ and $u^{u}=1 / \alpha$. One can readily find that the line $u^{u}=1 /(2 \alpha)$ is determined by the equation

$$
G_{m}=\frac{1}{2 \alpha^{2}}\left(1-\frac{2 \alpha \Delta}{3}\right)^{3 / 2} \quad \text { for } \quad \alpha \Delta<1 .
$$

This line is marked in Fig. $4 \mathrm{a}$ as $\Delta_{1}$. The line $u^{u}=1 / \alpha$ is determined by the equation

$$
G_{m}=\frac{1}{2 \alpha^{2}}\left[\left(1-\frac{2 \alpha \Delta}{3}\right)^{3 / 2}+1\right]-\frac{\Delta}{2 \alpha} \quad \text { for } \quad \alpha \Delta<0
$$

This line is marked in Fig. 4a as $\Delta_{2}$. Here the inequality $\alpha \Delta<0$ follows from the condition that, for $u=u^{u}=1 / \alpha$ to be a physical root of the cubic equation (2.25), the third (greatest) root of this equation must be greater than $1 / \alpha$. Note that (2.33) implies that for $\alpha \Delta=0$ one has $G_{m} \alpha^{2}=1$ so the line $\Delta_{2}$ starts at the upper boundary $\Delta_{+}^{\prime}$ of the existence of the hydraulic transcritical solution (see (2.29)).

In a similar way, for $\alpha<0$ we see that for the upstream flow one has $u^{-}=0$ and $u^{+}=u^{u}>0$, which corresponds to the 'normal' undular bore with the soliton of positive polarity at the leading edge (see Kamchatnov et al. (2012) and section 3 below). Downstream the obstacle three different regimes are possible depending on the value of $u^{d}$. The lines $u^{d}=1 /(2 \alpha)$ and $u^{d}=1 / \alpha$ separating the regions for different regimes on the solution map in Fig. $4 \mathrm{~b}$ are marked as $\Delta_{1}$ and $\Delta_{2}$. Along the line $\Delta_{2}$ the third (smallest) root of the equation must (2.25) lie outside the segment $\left[u^{d}=1 / \alpha ; u^{u}\right]$, hence, we get $|\alpha| \Delta>0$ (cf. a similar restriction for $\alpha>0$ in (2.33)).

We stress that the universal transcritical flow-regime classifications above are constructed in terms of just two parameters $G_{m} \alpha^{2}$ and $|\alpha| \Delta$ while the original problem is specified by three parameters $\alpha, G_{m}$ and $\Delta$. This means that the same upstreamdownstream resolution pattern can be realised for two disparate flows characterised by the same values of $G_{m} \alpha^{2}$ and $|\alpha| \Delta$. Of course this property of transcritical stratified flows does not imply any non-uniqueness in the flow characterisation for a given fixed value of the stratification parameter $\alpha$ but could be useful in the experimental modelling where certain configurations are more accessible than others. 


\section{Dispersive regularisation of transcritical hydraulic jumps}

Before we proceed with the analytical description of the wave patterns generated in transcritical internal wave flows governed by the forced Gardner equation (1.1) we review the general classification of solutions to the initial step resolution (Riemann) problem described in our work, Kamchatnov et al. (2012). This will enable us to identify the particular solutions realised in the dispersive regularisation of the upstream and downstream hydraulic jumps forming in the transcritical flow. An important feature of these solutions is that, despite the unidirectional nature of the Gardner equation the solution of the step resolution problem generally consists of two parts (waves) which are pieced together. This is in sharp contrast with the KdV equation dynamics where the resolution of an initial step always occurs through a single wave (an undular bore or a rarefaction wave, see Gurevich \& Pitaevskii (1974)). The underlying mathematical reason behind this broader variety of solutions is the fact that the modulation system for the Gardner equation, unlike that for the $\mathrm{KdV}$ equation, is neither strictly hyperbolic (for $\alpha<0$ ) nor genuinely nonlinear (for both signs of $\alpha$ ), which brings a suggestive parallel with shock theory in hyperbolic conservation laws with non-convex flux (see e.g. LeFloch (2002)). The key components of the asymptotic wave patterns occurring in the step resolution problem for the Gardner equation are: undular bores (there are three different types of them), rarefaction waves and solibores.

\subsection{Undular bores: the "core" modulation solution}

We describe first the "core" modulation solution for slow variations of the parameters of the periodic wave solution (such as amplitude, frequency, mean value) in the undular bore solutions to the unforced Gardner equation. This modulation solution plays the key role in the analytical description of the upstream and downstream wave patterns. As we have already mentioned, the Gardner equation has several types of undular bore solutions realised for different sets of the initial step parameter values $u^{-}, u^{+}$. One of the main results of Kamchatnov et al. (2012) is that all modulation solutions for the Gardner equation 'cnoidal' undular bores can be mapped onto the same classical Gurevich-Pitaevskii modulation solution for the KdV equation (see Gurevich \& Pitaevskii (1974), Fornberg \& Whitham (1978)). We would like to stress that this mapping is exact and is valid within the entire domain of the existence of the relevant undular bore solutions and thus, is different from an approximate near-identity transformation used in Marchant \& Smyth (1990) to describe the weakly nonlinear transcritical regimes of forced Gardner equation when the higher nonlinear term is small and the upstream and downstream undular bore solutions are asymptotically close to those of the KdV equation.

The mapping between the Gardner and the KdV modulation solutions is, however, not one-to-one so the "physical" modulations in the Gardner undular bores are reconstructed using one of the inverse mappings between the parameters of the relevant periodic solution of the Gardner equation and the modulation variables in the Gurevich-Pitaevskii solution. The choice of the inverse mapping is determined by the sign of $\alpha$ and the position of the initial step parameters $u^{-}, u^{+}$relative to the turning point $u=1 /(2 \alpha)$ of the dispersionless limit velocity $c(u)=\Delta-6 u(1-\alpha u)$ of the Gardner equation (1.1).

Another distinctive feature of the Gardner modulation dynamics is that for $\alpha<0$ it admits a special type of undular bores, termed nonlinear trigonometric bores, which have no analogue in the Gurevich-Pitaevskii theory for the KdV equation. These trigonometric undular bore solutions were first found in the focusing modified KdV equation (Marchant (2008)) and complex modified KdV equation (Kodama et al. (2008)). A detailed description of trigonometric bores for the Gardner equation was made in Kamchatnov et al. (2012). 
All periodic solutions of the Gardner equation satisfy the ordinary differential equation (ODE)

$$
u_{\xi}^{2}=\alpha\left(u-u_{1}\right)\left(u-u_{2}\right)\left(u-u_{3}\right)\left(u-u_{4}\right),
$$

where $\xi=x-V t-\xi_{0}, V$ being the phase velocity and $\xi_{0}$ an arbitrary initial phase (see Kamchatnov et al. (2012) for details). In the modulationally stable case of our interest the roots of the polynomial in the right-hand side of (3.1) are ordered

$$
u_{1} \leq u_{2} \leq u_{3} \leq u_{4}
$$

and satisfy the condition

$$
\sum_{i=1}^{4} u_{i}=\frac{2}{\alpha}
$$

so only three of them are independent. It is still convenient to keep all four $u_{j}$ 's in the subsequent formulae to preserve symmetry of the expressions. The phase velocity $V$ is expressed in terms of $u_{j}$ 's as

$$
V=\Delta-\alpha\left(u_{1} u_{2}+u_{1} u_{3}+u_{1} u_{4}+u_{2} u_{3}+u_{2} u_{4}+u_{3} u_{4}\right) .
$$

Now, if the parameters $u_{j}$ of the periodic solution are allowed to vary slowly with $x, t$, one arrives at a set of constraints on their variations, which have the form of quasilinear partial differential equations called the Whitham modulation equations (Whitham (1965, 1974)). The Whitham equations can be obtained by averaging conservations laws of the dispersive evolution equation over the periodic solution family.

It is clear that the actual form of the periodic solution of the Gardner equation specified by ODE (3.1) will depend on the sign of $\alpha$ and on the interval $\left(u_{i}, u_{i+1}\right)$ within which the variable $u$ oscillates. As a result, for each family of the periodic solutions the Whitham averaging procedure would yield its 'own' modulation system for $\left\{u_{j}(x, t), j=1, \ldots, 4\right\}$ (we recall that only three of $u_{j}$ 's are independent so the modulation system will consist of three equations). However, as was shown in Kamchatnov et al. (2012), for each set $\left\{u_{j}(x, t)\right\}$, there is a mapping $\left\{u_{1}, u_{2}, u_{3}, u_{4}\right\} \mapsto\left\{r_{1}, r_{2}, r_{3}\right\}$, where $r_{3}(x, t) \geq r_{2}(x, t) \geq$ $r_{1}(x, t)$ are the Riemann invariants of the well-known KdV-Whitham modulation system (obtained by the Whitham averaging of the KdV reduction of the Gardner equation, when $\alpha=0$, see Whitham (1974)). This (quadratic) mapping has the form

$$
r_{1}=\frac{\alpha}{4}\left(u_{1}+u_{2}\right)\left(u_{3}+u_{4}\right), \quad r_{2}=\frac{\alpha}{4}\left(u_{1}+u_{3}\right)\left(u_{2}+u_{4}\right), \quad r_{3}=\frac{\alpha}{4}\left(u_{2}+u_{3}\right)\left(u_{1}+u_{4}\right)
$$

for $\alpha>0$ and

$$
r_{1}=\frac{\alpha}{4}\left(u_{1}+u_{4}\right)\left(u_{2}+u_{3}\right), \quad r_{2}=\frac{\alpha}{4}\left(u_{1}+u_{3}\right)\left(u_{2}+u_{4}\right), \quad r_{3}=\frac{\alpha}{4}\left(u_{1}+u_{2}\right)\left(u_{3}+u_{4}\right)
$$

for $\alpha<0$.

The similarity modulation solution of the $\mathrm{KdV}$-Whitham system describing the dependence of $r_{j}$ 's on $x$ and $t$ in the undular bore is well known (Gurevich \& Pitaevskii (1974), Fornberg \& Whitham (1978)) and has the form :

$$
r_{1}=r^{-}, \quad r_{3}=r^{+},
$$

where $r^{+}>r^{-}$are constants, whereas the function $r_{2}(x, t)$, with an account of the coefficients in the original Gardner equation (1.1), is given implicitly by

$$
\Delta-2\left(r_{1}+r_{2}+r_{3}\right)+\frac{4\left(r_{2}-r_{1}\right)(1-m) \mathrm{K}(m)}{\mathrm{E}(m)-(1-m) \mathrm{K}(m)}=\frac{x}{t},
$$




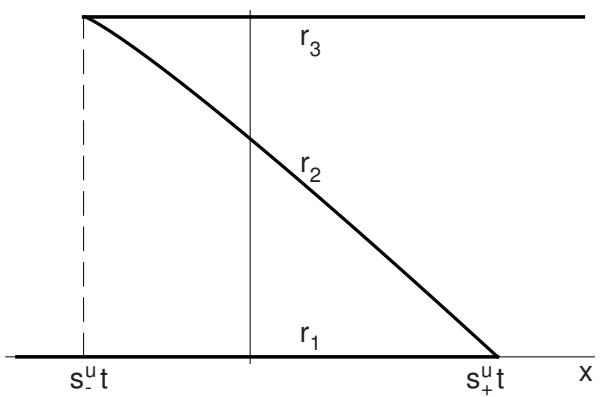

Figure 6. Typical dependence of the Riemann invariants $r_{i}$ on the space coordinate $x$ in the Gurevich-Pitaevskii modulation solution (3.7), (3.8) for $r^{-}=0, r^{+}>0$ at some $t>0$.

where $\mathrm{K}(m)$ and $\mathrm{E}(m)$ are the complete elliptic integrals of the fist and second kind respectively, and the modulus

$$
m=\frac{r_{2}-r_{1}}{r_{3}-r_{1}}=\frac{\left(u_{3}-u_{2}\right)\left(u_{4}-u_{1}\right)}{\left(u_{4}-u_{2}\right)\left(u_{3}-u_{1}\right)} .
$$

The constants $r_{1}=r^{-}, r_{3}=r^{+}$are determined by the initial step parameters $u^{-}, u^{+}$. These values also determine the specific set of relationships $u_{j}(\mathbf{r}$ ) (one of the inverses to (3.5) or (3.6)) to be used in the reconstruction of the Gardner undular bore solution from the dependence (3.8) (see Kamchatnov et al. (2012)).

The modulated wavetrain described by $(3.7),(3.8)$ is confined to an expanding region

$$
s^{-} t<x<s^{+} t
$$

where the leading and trailing edge speeds are

$$
s^{-}=\Delta-2 r^{-}-4 r^{+}, \quad s^{+}=\Delta-12 r^{-}+6 r^{+}
$$

respectively. The values $s^{ \pm}$are found by substituting of $m=1$ (soliton edge $x=s^{-} t$ ) and $m=0$ (small amplitude edge $x=s^{+} t$ ) in the similarity solution (3.8). The behaviour of the Riemann invariants $r_{j}(x, t)$ in the "core" modulation solution (3.7), (3.8) is shown in Fig. 6. The inverse mappings $\left\{r_{1}, r_{2}, r_{3}\right\} \mapsto\left\{u_{1}, u_{2}, u_{3}, u_{4}\right\}$ for each relevant case will be described separately in sections 4 and 5 .

\subsection{Riemann problem classification}

To describe the upstream and downstream closure of the localised transcritical transition described in section 2 we need to know solutions of the Gardner equation (1.1) with zero forcing term and steplike initial conditions (2.31). This (Riemann) problem was considered in Kamchatnov et al. (2012); here we present the resulting classifications of the solutions for $\alpha>0$ and $\alpha<0$. Note that in Kamchatnov et al. (2012) the Gardner equation was taken in the standard form

$$
\tilde{u}_{\tilde{t}}+6 \tilde{u} \tilde{u}_{\tilde{x}}-6 \alpha \tilde{u}^{2} \tilde{u}_{\tilde{x}}+\tilde{u}_{\tilde{x} \tilde{x} \tilde{x}}=0,
$$

which is related to the equation (1.1) with $G_{m} \equiv 0$ by a simple linear transformation $u=\tilde{u}, \quad t=\tilde{t}, \quad x=-\tilde{x}+\Delta \tilde{t}$. The step conditions for (3.12) corresponding to (2.31) are

$$
\tilde{u}(\tilde{x}, 0)= \begin{cases}u^{+}, & \tilde{x}<0 \\ u^{-}, & \tilde{x}>0 .\end{cases}
$$

The classifications of solutions to the Riemann problem (3.12), (3.13) with $\alpha>0$ and 


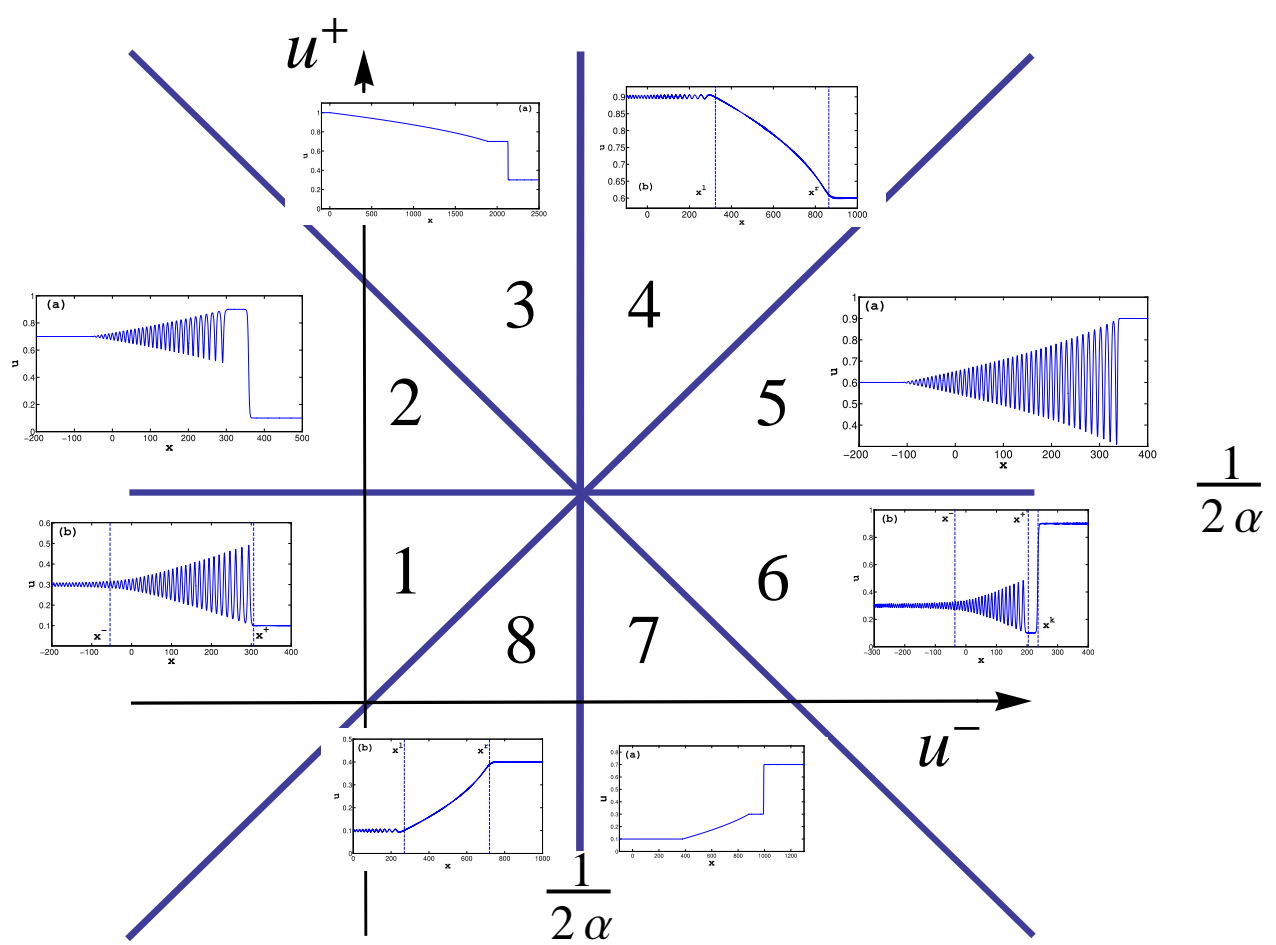

FiguRE 7. (Color online) Parametric map of solutions to the Gardner equation (3.12) with $\alpha>0$ and initial step conditions (3.13) (from Kamchatnov et al. (2012)). The resolution diagrams corresponding to each of the cases on the plane of the initial step parameters $u^{+}$and $u^{-}$are the following: Region 1: $\left\{u^{+} \mathbf{U B} \rightarrow u^{-}\right\}$; Region 2: $\left\{u^{+} \leftarrow \mathbf{U B}\left(\tilde{u}^{*}\right)\right.$ SB $\left.\rightarrow u^{-}\right\}$; Region 3: $\left\{u^{+} \leftarrow \mathbf{R W}\left(\tilde{u}^{*}\right) \mathbf{S B} \rightarrow u^{-}\right\}$; Region 4: $\left\{u^{+} \leftarrow\right.$ RW $\left.u^{-}\right\}$; Region 5: $\left\{u^{+} \leftarrow\right.$ UB $\left.u^{-}\right\}$; Region 6: $\left\{u^{+}\right.$UB $\rightarrow\left(\tilde{u}^{*}\right) \leftarrow$ SB $\left.u^{-}\right\}$; Region $7:\left\{u^{+} \mathbf{R W} \rightarrow\left(\tilde{u}^{*}\right) \leftarrow\right.$ SB $\left.u^{-}\right\}$; Region 8: $\left\{u^{+}\right.$RW $\left.\rightarrow u^{-}\right\}$. In all relevant cases the intermediate state $\tilde{u}=\tilde{u}^{*}=1 / \alpha-u^{-}$.

$\alpha<0$ are presented in Fig. 7 and Fig. 8 respectively. Since in the transcritical flow we always have $u^{-}=0$ for the upstream solution and $u^{+}=0$ for the downstream solution, only certain parts of the full classification are relevant to the problem under study, these will be detailed in sections 4 and 5 . Here we only explain the notations for the step resolution diagrams used in the figure captions. The labels $\mathbf{U B}, \mathbf{R W}$ and $\mathbf{S B}$ denote the cnoidal undular bore, the rarefaction wave and the solibore respectively; the arrows denote the "normal" $(\rightarrow)$ and "reversed" $(\leftarrow)$ structure of the relevant wave pattern. The terms "normal" and "reversed" refer to the sign of the gradient of $\tilde{u}$ across the structure: in the "normal" undular bores and solibores the value of $\tilde{u}$ decreases when crossing the structure in the positive direction, while in the "normal" rarefaction wave this value increases. In the "reversed" structures the signs of the gradients are opposite to those in "normal" ones. A special feature of the reversed undular bores is that, despite the negative sign of linear dispersion in the Gardner equation, they exhibit "dark" solitons at their leading edges in contrast to the 'normal' KdV-type negative dispersion "bright soliton" pattern.

For instance, the diagram $\left\{u^{+} \leftarrow \mathbf{R W}\left(\tilde{u}^{*}\right) \mathbf{S B} \rightarrow u^{-}\right\}$in Fig. 7 (region 3) corresponds to the resolution pattern for the initial step (3.13) consisting of the "normal" solibore and the "reversed" rarefaction wave connected via an intermediate state $\tilde{u}^{*}=1 / \alpha-u^{-}$. The nonlinear trigonometric bores appearing in the classification for $\alpha<0$ are denoted as $\mathbf{T B}$. 


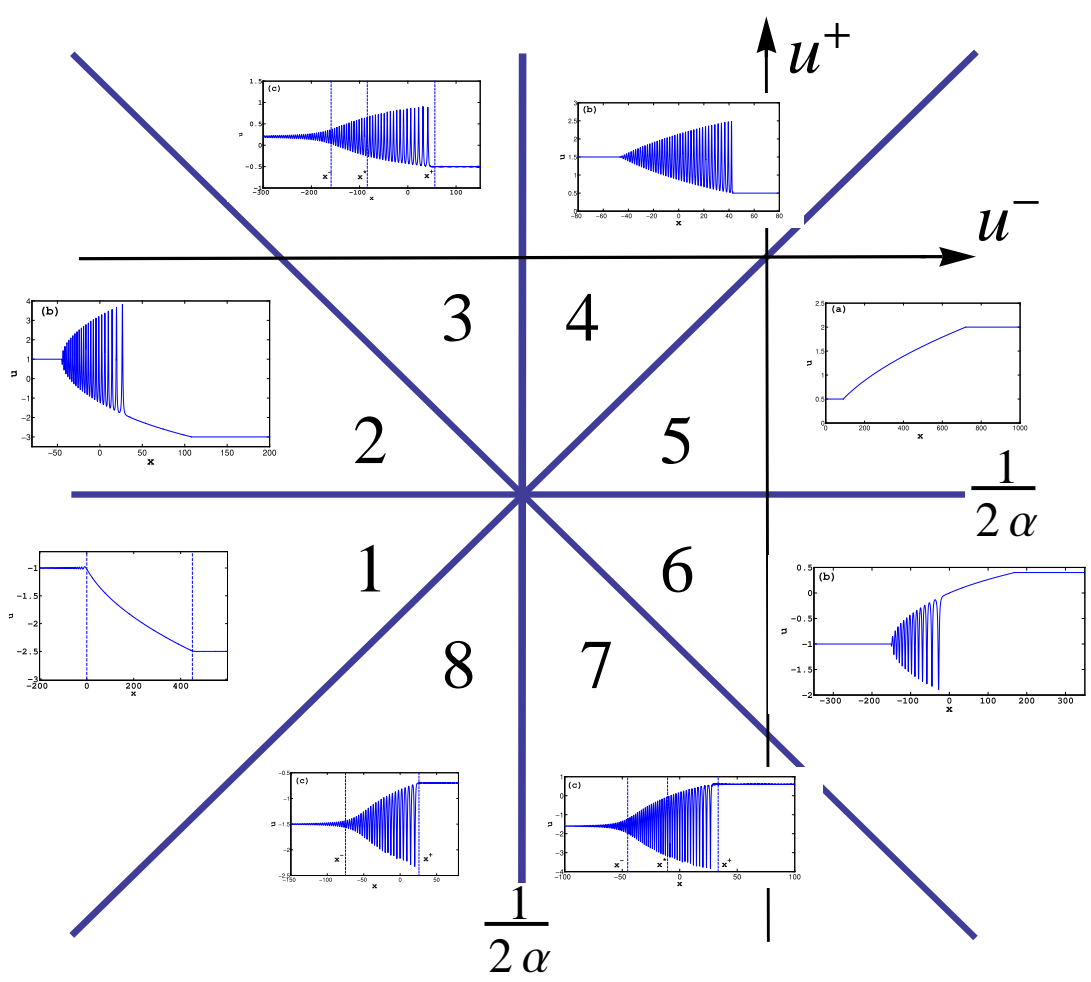

Figure 8. (Color online) Parametric map of solutions of the step problem for the Gardner equation (3.12) with $\alpha<0$ and initial step conditions (3.13) (from Kamchatnov et al. (2012)). The resolution diagrams corresponding to each of the cases on the plane of the initial step parameters $u^{-}$and $\tilde{u}^{+}$are the following: Region 1: $\left\{u^{+} \leftarrow \mathbf{R W} u^{-}\right\}$; Region $2:\left\{u^{+} \mathbf{T B} \rightarrow\left(\tilde{u}^{*}\right) \leftarrow \mathbf{R W} u^{-}\right\}$; Region 3: $\left\{\tilde{u}^{-}(\mathbf{T B} \mid \mathbf{U B}) \rightarrow \tilde{u}^{+}\right\}$; Region 4: $\left\{u^{+}\right.$UB $\left.\rightarrow u^{-}\right\}$; Region 5: $\left\{u^{+} \mathbf{R W} \rightarrow\right.$ $\left.u^{-}\right\}$; Region 6: $\left\{u^{+} \leftarrow\right.$ TB $\left.\left(\tilde{u}^{*}\right) \mathbf{R W} \rightarrow u^{-}\right\}$; Region $7:\left\{u^{+} \leftarrow\right.$ (TB|UB) $\left.u^{-}\right\}$; Region 8: $\left\{u^{+} \leftarrow \mathbf{U B} u^{-}\right\}$. In all relevant cases the intermediate state $\tilde{u}=\tilde{u}^{*}=1 / \alpha-u^{+}$.

In a number of cases, the trigonometric bore occurs as part of a composite structure: say, the resolution diagram $\left\{u^{+} \leftarrow\right.$ (TB|UB) $\left.u^{-}\right\}$in Fig. 8 (Region 7 ) denotes the composite cnoidal-trigonometric undular bore connecting the constant boundary states $u^{-}$and $u^{+}$.

\section{Upstream and downstream closure, $\alpha>0$}

\subsection{Upstream solution}

To describe the upstream wave pattern we need to solve the unforced Gardner equation (1.1) with initial conditions (2.31) where $u^{-}=0$ and $u^{+}=u^{u}$. The dependence of the upstream hydraulic elevation $u^{u}$ on the parameters $\Delta, G_{m}$ is given by $(2.25),(2.26)$. The value of $u^{u}$ within the region of existence of the localised transcritical hydraulic solution (see Fig. 5a) ranges from $\left(u^{u}\right)_{\min }=0$ at the lower boundary $\Delta_{-}$to some $\left(u^{u}\right)_{\max }$ at the upper boundary $\Delta_{+}^{\prime}$. The value $\left(u^{u}\right)_{\max }$ is found as the smaller positive root of equation $(2.25)$ with $G_{m}(\Delta)$ defined by $(2.29)$.

An inspection of the classification presented in Fig. 7 shows that within this range of $u^{+}=u^{u}$ (at fixed $u^{-}=0$ ) one can possibly have three different types of the solution corresponding to Regions 1,2 and 3. These solutions are presented below. 
(a) $\Delta_{-}<\Delta<\Delta_{1}$ (see Fig. 4a). This corresponds to $0<u^{u}<1 /(2 \alpha)$ and, hence, to Region 1 in the classification map in Fig. 7. Thus, we have an upstream propagating undular bore with the bright soliton at the leading (left) edge (note the change in the propagation direction when applying the classification in Fig. 7 to equation (1.1)). The modulation solution for this bore is given by (3.7), (3.8), where the constant Riemann invariants (3.7) are

$$
r_{1}=0, \quad r_{3}=u^{u}\left(1-\alpha u^{u}\right),
$$

and the dependence of the varying Riemann invariant $r_{2}$ on $x$ and $t$ is given in an implicit form by (3.8).

The oscillatory structure of the undular bore is described by the periodic solution of the ODE (3.1), which is expressed in terms of the Jacobi elliptic functions as (see Kamchatnov et al. (2012))

$$
u=u_{2}+\frac{\left(u_{3}-u_{2}\right) \mathrm{cn}^{2}(\theta, m)}{1-\frac{u_{3}-u_{2}}{u_{4}-u_{2}} \operatorname{sn}^{2}(\theta, m)},
$$

where the phase is

$$
\theta=\sqrt{\alpha\left(u_{3}-u_{1}\right)\left(u_{4}-u_{2}\right)}\left(x-x_{0}-V t\right) / 2,
$$

the modulus $m$ is given by (3.9), and the phase velocity $V$ is

$$
V=\Delta-\alpha\left(u_{1} u_{2}+u_{1} u_{3}+u_{1} u_{4}+u_{2} u_{3}+u_{2} u_{4}+u_{3} u_{4}\right) .
$$

In the small-amplitude limit $u_{3} \rightarrow u_{2}\left(m_{1} \rightarrow 0\right)$ we get the linear harmonic wave

$$
\begin{aligned}
& u \cong u_{2}+\frac{1}{2}\left(u_{3}-u_{2}\right) \cos \left[k\left(x-x_{0}-V t\right)\right], \\
& k=\sqrt{\alpha\left(u_{2}-u_{1}\right)\left(u_{4}-u_{2}\right)}, \\
& V=\Delta-4 u_{2}-\alpha\left(u_{1} u_{4}-3 u_{2}^{2}\right) .
\end{aligned}
$$

The soliton limit $m \rightarrow 1$ can be achieved in one of the two ways: when $u_{1} \rightarrow u_{2}$ or when $u_{3} \rightarrow u_{4}$. In the Region 1 the soliton limit occurs via $u_{2} \rightarrow u_{1}$ (see Kamchatnov et al. (2012)) so we obtain a "bright" soliton of elevation propagating against a constant background $u=u_{1}$,

$$
u(\theta)=u_{1}+\frac{u_{3}-u_{1}}{\cosh ^{2} \theta-\frac{u_{3}-u_{1}}{u_{4}-u_{1}} \sinh ^{2} \theta} .
$$

If, further, one has $u_{4}-u_{3} \ll u_{3}-u_{2}$, then the soliton (4.6) becomes a wide, "table-top" soliton.

For Region 1, the expressions for the parameters $u_{j}$ in terms of the Riemann invariants $r_{1}, r_{2}, r_{3}$ are given by the following inverse formulae of (3.6) (see Kamchatnov et al. (2012)),

$$
\begin{aligned}
& u_{1}=\frac{1}{2 \alpha}\left(1-\sqrt{1-4 \alpha r_{1}}-\sqrt{1-4 \alpha r_{2}}+\sqrt{1-4 \alpha r_{3}}\right), \\
& u_{2}=\frac{1}{2 \alpha}\left(1-\sqrt{1-4 \alpha r_{1}}+\sqrt{1-4 \alpha r_{2}}-\sqrt{1-4 \alpha r_{3}}\right), \\
& u_{3}=\frac{1}{2 \alpha}\left(1+\sqrt{1-4 \alpha r_{1}}-\sqrt{1-4 \alpha r_{2}}-\sqrt{1-4 \alpha r_{3}}\right), \\
& u_{4}=\frac{1}{2 \alpha}\left(1+\sqrt{1-4 \alpha r_{1}}+\sqrt{1-4 \alpha r_{2}}+\sqrt{1-4 \alpha r_{3}}\right),
\end{aligned}
$$

which, after the substitution of (4.1), assume the form (note that $\left(1-4 \alpha r_{3}\right)^{1 / 2}=1-2 \alpha u^{u}$ 

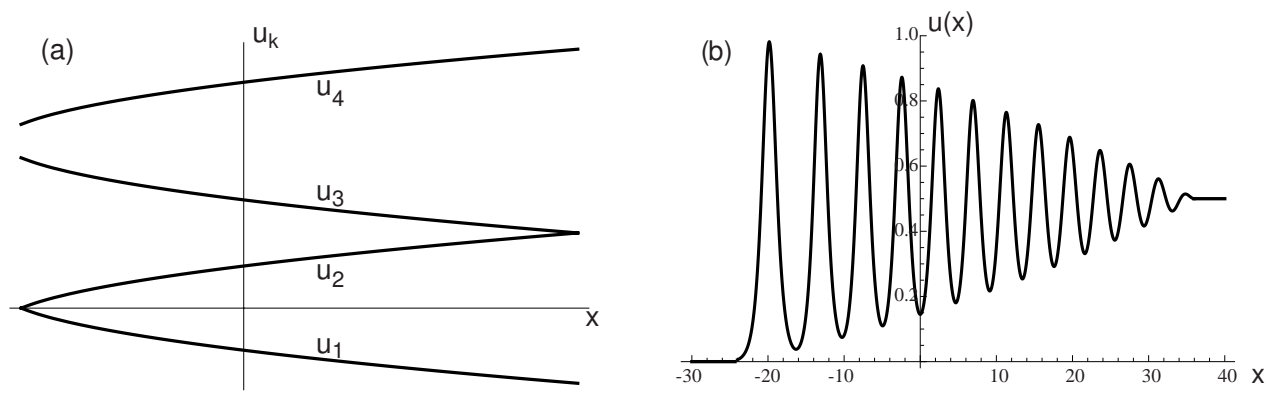

Figure 9. Riemann problem solution to the unforced Gardner equation (1.1) with $\alpha>0$ and the initial step parameters $u^{-}=0, u^{+}=u^{u}, 0<u^{u}<1 /(2 \alpha)$, corresponding to the upstream resolution of the transcritical flow via a normal undular bore (Region 1 in the Riemann problem classification in Fig. 7 and the region $\Delta_{-}<\Delta<\Delta_{1}$ of the transcritical flow-regime diagram in Fig. 4a). (a) Typical behaviour of the modulation parameters $u_{k}$ in the undular bore. At the soliton edge $u_{1}=u_{2}=0$; at the linear edge $u_{2}=u_{3}=u^{u}$; (b) Plot of the analytical (modulation theory) solution to the Gardner equation with $\alpha=1, \Delta=0$ and the step-like initial condition (2.31) with $u^{-}=0, u^{+}=0.45$ at the time $t=40$. Only the upstream part, $x<0$, of the bore is realised in the transcritical flow.

for $0<u^{u}<1 /(2 \alpha)-$ see $\left.(4.1)\right)$ :

$$
\begin{aligned}
& u_{1}\left(r_{2}\right)=\frac{1}{2 \alpha}\left(1-2 \alpha u^{u}-\sqrt{1-4 \alpha r_{2}}\right), \\
& u_{2}\left(r_{2}\right)=\frac{1}{2 \alpha}\left(-1+2 \alpha u^{u}+\sqrt{1-4 \alpha r_{2}}\right), \\
& u_{3}\left(r_{2}\right)=\frac{1}{2 \alpha}\left(1+2 \alpha u^{u}-\sqrt{1-4 \alpha r_{2}}\right), \\
& u_{4}\left(r_{2}\right)=\frac{1}{2 \alpha}\left(3-2 \alpha u^{u}+\sqrt{1-4 \alpha r_{2}}\right) .
\end{aligned}
$$

One can see that the limit $m=1$ (i.e. $r_{2}=r_{3}=u^{u}\left(1-\alpha u^{u}\right)$ ) is indeed achieved via $u_{2}=u_{1}=0$ (see (3.9)), which corresponds to the "bright" soliton (4.6). In the linear limit $m=0\left(r_{2}=r_{1}=0\right)$ one has $u_{3}=u_{4}=u^{u}$. The qualitative behaviour of $u_{j}$ 's (4.8) generated by the "core" solution (3.8) in the normal undular bore (Region 1 in Fig. 7) is shown in Fig. 9a.

The speeds of the leading (soliton) and the trailing (linear) edges of the upstream undular bore edges are given by (see (3.11), (4.1))

$$
s_{u}^{-}=\Delta-4 u^{u}\left(1-\alpha u^{u}\right), \quad s_{u}^{+}=\Delta+6 u^{u}\left(1-\alpha u^{u}\right)
$$

respectively. Formulae (4.9), via $u^{u}\left(\Delta, G_{m}\right)$ specified by (2.25), (2.26) define the undular bore edge speeds in terms of the input parameters $\Delta, G_{m}$. The amplitude of the lead soliton is $a^{-}=2 u^{u}$.

It is clear that the upstream undular bore can be realised fully only if its trailing edge propagates upstream, i.e. if $s^{+}<0$. The condition $s^{+}=0$ yields then the equation of the line in the $\left(\alpha \Delta, G_{m} \alpha^{2}\right)$-plane of the flow-regime diagram (see Fig. 4a) corresponding to the generation of an upstream undular bore whose zero-amplitude trailing edge is at rest at the obstacle's location (effectively at $x=0$ ). Since $u=u^{u}$ is a solution of (2.25), an analytical expression for this line $\Delta=\Delta^{u}\left(G_{m}\right)$ is obtained by finding $u^{u}$ from the 


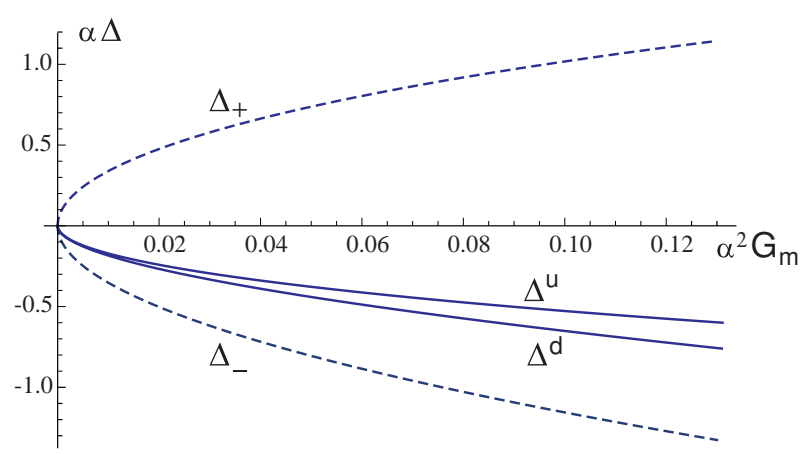

FiguRE 10. Detailed structure of the transcritical flow-regime diagram for $\alpha \Delta<1$. The lines $\Delta^{u}$ and $\Delta^{d}$ in the $\left(G_{m} \alpha^{2}, \Delta \alpha\right)$-plane separate the sub-regions of the parameters corresponding to the generation of the attached (partial) and detached (fully realised) bores. The line $\Delta^{u}$ is specified by (4.10), the line $\Delta^{d}$ is specified by (4.21). The boundaries $\Delta_{ \pm}$of the transcritical region are shown by dashed lines. Specifically: (i) $\Delta_{-}<\Delta<\Delta^{d}-$ the upstream bore is detached, the downstream bore is attached; (ii) $\Delta^{d}<\Delta<\Delta^{u}$ - both bores are detached; (iii) $\Delta^{u}<\Delta<\Delta_{+}$- the upstream bore is attached, the downstream bore is detached. For $\alpha \Delta>1$ the upper transcritical boundary $\Delta_{+}$in (iii) should be replaced by $\Delta_{1}-$ see Fig. 4 a.

equation $s^{+}=0$ specified by (4.9) and then substituting it into (2.25):

$\Delta^{u}: \quad G_{m} \alpha^{2}=\frac{1}{2}\left\{\frac{1}{2}\left(1-\sqrt{1+\frac{2 \alpha \Delta}{3}}\right)\left(1-\sqrt{1+\frac{2 \alpha \Delta}{3}}-\frac{8 \alpha \Delta}{3}\right)-1+\alpha \Delta+\left(1-\frac{2 \alpha \Delta}{3}\right)^{3 / 2}\right\}$.

Expanding (4.10) for small $|\alpha \Delta| \ll 1$ we obtain:

$$
\Delta^{u}: \quad G_{m} \alpha^{2} \cong \frac{1}{3}(\alpha \Delta)^{2}-\frac{1}{27}(\alpha \Delta)^{3} .
$$

The inverse function $\Delta\left(G_{m}\right)$ to (4.10) has two branches; we are interested in the lower one located within the region $\Delta_{-}<\Delta<\Delta_{1}$. Above this line one has $s^{+}>0$, and, therefore, the upstream undular bore can be realised only partially in this region of the input parameters (see Fig. 10). We also note that the leading term of the expansion (4.11) $\Delta=-\sqrt{3 G_{m}}$ agrees with the forced KdV result by Grimshaw \& Smyth (1986) and Smyth (1987), as expected. We also note that the separation curve (4.10) is defined only for $\alpha \Delta>-3 / 2$ (the existence of the real root $u^{u}$ of the quadratic equation $s^{+}=0$ ). For $\alpha \Delta<-3 / 2$ the upstream bore is always detached.

An example of the Riemann problem solution (4.2), (4.8), (3.7), (3.8) corresponding to the upstream flow resolution via partial bore is shown in Fig. 9b. Indeed, in the context of the transcritical flow, the downstream part of the bore cannot be realised and one ends up with the partial undular bore attached to the obstacle at $x=0$ (as long as the modulation description is concerned). The partial undular bore has some nonzero value of the modulus $m=m_{0}>0$ (and, therefore, some non-zero amplitude $a=a_{0}>0$ ) at the attachment point. The parameters $m_{0}$ and $a_{0}$ are found from the similarity solution (3.8) with $r_{1}=0$ complemented by the condition of continuity for the mean value $\bar{u}$ at $x=0$, where one requires $\bar{u}=u^{u}$. The analysis is equivalent to that performed in Grimshaw \& Smyth (1986) and Smyth (1987) for the forced KdV equation so we do not present it here. We just mention that, if $m_{0}$ is sufficiently close to unity one can replace the partial undular bore by a uniform solitary wave train.

(b) $\Delta_{1}<\Delta<\Delta_{2}$ (see Fig. 4a). This corresponds to $1 /(2 \alpha)<u^{u}<1 / \alpha$ and, hence, 

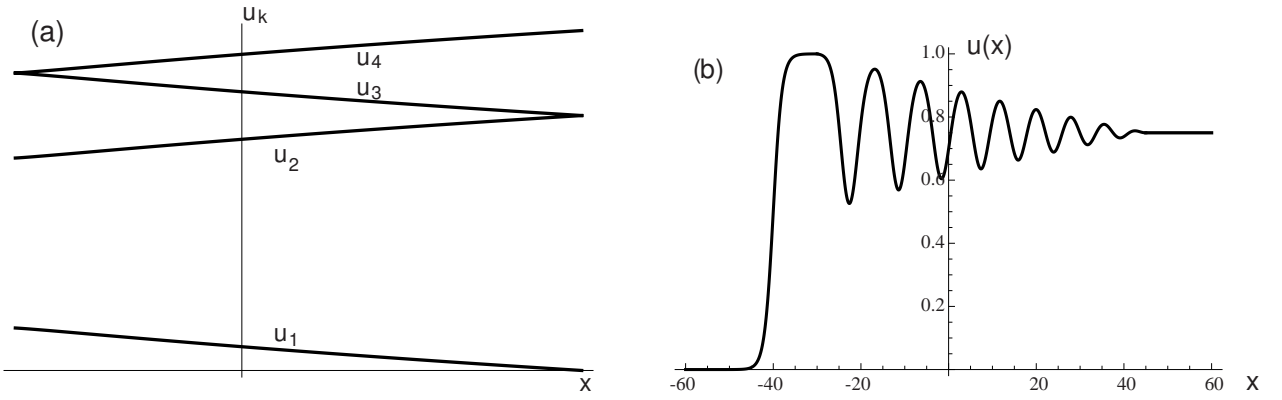

FIGURE 11. Riemann problem solution to the unforced Gardner equation (1.1) with $\alpha>0$ and the initial step parameters $u^{-}=0, u^{+}=u^{u}, 1 /(2 \alpha)<u^{u}<1 / \alpha$, corresponding to the region $\Delta_{1}<\Delta<\Delta_{2}$ in the transcritical flow diagram in Fig. 4a (Region 2 in Riemann problem classification in Fig 7). (a) Typical behaviour of the modulation parameters $u_{k}$ in the reversed undular bore. At the leading (dark soliton) edge of the bore $u_{3}=u_{4}=u^{*}=1 / \alpha$; at the trailing (linear) edge $u_{3}=u_{2}=u^{u}<1 / \alpha$. (b) Plot of the analytical (modulation theory) solution to the Gardner equation with $\alpha=1, \Delta=0$ and the step-like initial condition (2.31) with $u^{-}=0$, $u^{+}=0.75$ at the time $t=40$. The constant state $u=u^{*}=1$ at the leading edge of the reversed undular bore is further connected with the equilibrium flow upstream $u=0$ via a solibore.

to the Region 2 in the parametric map of the Riemann problem in Fig. 7. The upstream wave structure now consists of the reversed undular bore with the dark soliton at the leading edge and a solibore propagating to the left into the undisturbed state $u^{-}=0$ (see Fig. 11). The undular bore and the solibore are joined together via an intermediate state $u=u^{*}=1 / \alpha-u^{-}$.

The reversed undular bore is described by the same 'core' modulation solution (4.1), (3.8) in terms of the Riemann invariants $r_{j}, j=1,2,3$ but the relationships between the parameters $\left\{u_{j}\right\}$ in the periodic solution (4.2) and the Riemann invariants $\left\{r_{k}\right\}$ are now given by the second set of the inverse formulae to (3.5),

$$
\begin{aligned}
& u_{1}=\frac{1}{2 \alpha}\left(1-\sqrt{1-4 \alpha r_{1}}-\sqrt{1-4 \alpha r_{2}}-\sqrt{1-4 \alpha r_{3}}\right), \\
& u_{2}=\frac{1}{2 \alpha}\left(1-\sqrt{1-4 \alpha r_{1}}+\sqrt{1-4 \alpha r_{2}}+\sqrt{1-4 \alpha r_{3}}\right), \\
& u_{3}=\frac{1}{2 \alpha}\left(1+\sqrt{1-4 \alpha r_{1}}-\sqrt{1-4 \alpha r_{2}}+\sqrt{1-4 \alpha r_{3}}\right), \\
& u_{4}=\frac{1}{2 \alpha}\left(1+\sqrt{1-4 \alpha r_{1}}+\sqrt{1-4 \alpha r_{2}}-\sqrt{1-4 \alpha r_{3}}\right) .
\end{aligned}
$$

Upon substituting (4.1) and using that $\left(1-4 \alpha r_{3}\right)^{1 / 2}=2 \alpha u^{+}-1$ (now $u^{+}>1 /(2 \alpha)$ ), one can readily see that the relationships (4.12) assume the same form (4.8). However, due to the different sign of $\left(1-4 \alpha r_{3}\right)^{1 / 2}$, the soliton limit $r_{2}=r_{3}(m=1)$ is now achieved via $u_{3}=u_{4}$, which corresponds to the dark soliton limit of the periodic solution (4.2) (see Kamchatnov et al. (2012) for details):

$$
u(\theta)=u_{4}-\frac{u_{4}-u_{2}}{\cosh ^{2} \theta-\frac{u_{4}-u_{2}}{u_{4}-u_{1}} \sinh ^{2} \theta} .
$$

Now, it follows from (4.12) that, when $r_{2}$ varies in the interval $r_{1} \leq r_{2} \leq r_{3}$ according to (3.8), the parameters $u_{k}$ change along the curves shown in Fig. 11a (cf. Fig. 9a for the Region 1 undular bore). The left degeneration point $u_{3}=u_{4}=1 / \alpha$ corresponds to a dark soliton (4.13) riding on the background $u=u^{*}=1 / \alpha>0$, while at the opposite edge we have $u_{3}=u_{2}=u^{+}=u^{u}$, which corresponds to a linear wave packet propagating 


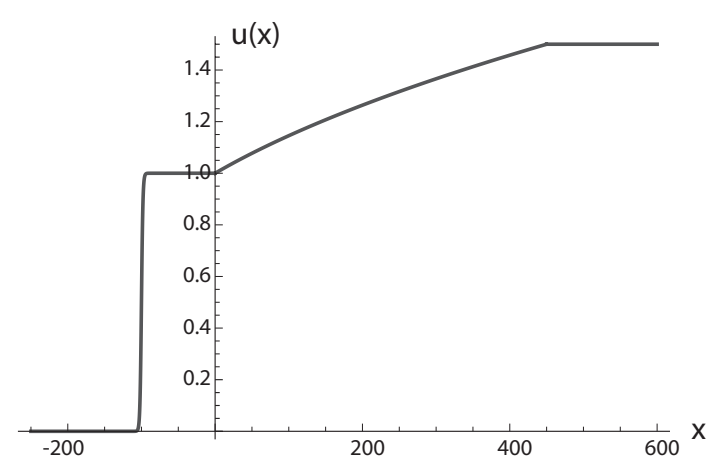

Figure 12. Asymptotic solution (4.14), (4.15) to the Riemann problem for the unforced Gardner equation (1.1) with $\alpha>0$ and the initial step parameters $u^{-}=0, u^{+}=u^{u}$, where $1 / \alpha<u^{u}<u^{u}\left(\Delta_{+}^{\prime}\right)$ (Region 3 in the Riemann problem classification Fig 7 and the corresponding region $\Delta_{2}<\Delta<\Delta_{+}^{\prime}$ in the transcritical flow-regime diagram in Fig. 4a). The pattern consists of the rarefaction wave (exact solution of the dispersionless Gardner equation) and the solibore joined together via the constant state $u=1 / \alpha$. The plot parameters are: $\alpha=1, \Delta=0$, $u^{-}=0, u^{+}=u^{u}=1.5, t=100$.

on the uniform background $u=u^{u}$. Thus, substitution of $u_{k}(x, t)$ in the periodic solution (4.2) yields the reversed undular bore connecting the levels $u=u^{u}$ and $u=1 / \alpha$ (see Fig. 11b). The lead soliton amplitude in this undular bore is $a^{*}=2\left(1 / \alpha-u^{u}\right)$.

The intermediate state $u=1 / \alpha$ at the leading edge is further connected with the upstream equilibrium state $u=0$ by a solibore, which is obtained as the limiting form of the periodic solution (4.2) when $u_{2} \rightarrow u_{1}$ and $u_{3} \rightarrow u_{4}$ simultaneously. There are two possible solibore solutions, the one relevant to Region 2 has the form

$$
u(x, t)=\frac{1}{\alpha}\left(1-\frac{1}{\exp \left[\sqrt{\alpha}\left(x-s^{k} t\right)\right]+1}\right), \quad s^{k}=\Delta-1 / \alpha .
$$

The type of the initial step resolution via the combination of an undular bore and a solibore described above is sometimes referred to as the dispersive "lock-exchange" (in contrast to the dispersive "dam-break" type resolution in Region 1, see for instance Esler \& Pierce (2011). The speeds of the soliton and linear edges of the reversed upstream undular bore in the lock-exchange solution are given by the same formulae (4.9) which, for the region $\Delta_{1}<\Delta<\Delta_{2}$ considered, implies that the reversed bore is attached to the obstacle and realised only partially as long as $\alpha \Delta>-3 / 2$ (see (4.10)). We note that, although this pattern can be seen in the previous numerical simulations (see e.g. Grimshaw et al. (2002)) it had not been identified as a separate distinct feature of the dispersive transcritical stratified flows.

(c) $\Delta_{2}<\Delta<\Delta_{+}^{\prime}$ (see Fig. 4a; note that this narrow region is located entirely in the lower half-plane, i.e. for $\left.\alpha \Delta<0, G_{m} \alpha^{2}>1\right)$. This corresponds to $1 / \alpha<u^{u}<u^{u}\left(\Delta_{+}^{\prime}\right)$ and, hence, to the Region 3 in the parametric classification of the Riemann problem in Fig. 7. The upstream wave structure now consists of the combination of the solibore and the reversed rarefaction wave connected via an intermediate state $u=u^{*}=1 / \alpha$. This is the classical lock-exchange pattern observed in previous numerical simulations of the forced Gardner equation (see e.g. Melville \& Helfrich (1987), Grimshaw et al. (2002)) and also in fully nonlinear two-layer transcritical flows White \& Helfrich (2012). The solibore connecting the equilibrium state $u=0$ upstream and the intermediate constant state $u=1 / \alpha$ is described by formula (4.14). The constant state is further connected with the upstream hydraulic elevation state $u=u^{u}$ via the reversed simple rarefaction 
wave, which is asymptotically described by the similarity solution of the dispersionless limit of the unforced Gardner equation,

$$
\begin{aligned}
& u=1 / \alpha, \text { for } x<s^{l} t, \\
& u=\frac{1}{2 \alpha}\left(1+\sqrt{1+\frac{2 \alpha}{3}\left(\frac{x}{t}-\Delta\right)}\right) \text { for } s^{l} t<x<s^{r} t, \\
& u=u^{u} \text { for } \quad x>s^{r} t,
\end{aligned}
$$

where the edge speeds are

$$
s^{l}=\Delta, \quad s^{r}=\Delta-6 u^{u}\left(1-\alpha u^{u}\right) .
$$

As a matter of fact, $s^{k}<s^{l}$ (see (4.14)). Also, if $s^{r}>0$, the upstream structure is realised only partially. The combined solution (4.14), (4.15) is shown in Fig. 12. The weak discontinuities at the corners of the hydrodynamic rarefaction wave are resolved by small-amplitude dispersive wavetrains which asymptotically decay with time.

\subsection{Downstream solution}

The dispersive resolution of the downstream hydraulic jump occurs between the states $u^{-}=u^{d}<0$, and $u^{+}=0$. An inspection of the classification presented in Fig. 7 shows that for this range of $u^{+}, u^{-}$the only possible solution is the normal cnoidal undular bore corresponding to Region 1 in the Riemann problem classification in Fig. 7.

The oscillatory structure of the bore is described by the elliptic solution (4.2) with the parameters $u_{j}, j=1, \ldots, 4$ expressed in terms of the Riemann invariants $r_{i}, i=1,2,3$ via relationships (4.7). The spatiotemporal behaviour of the Riemann invariants is given by the Gurevich-Pitaevskii similarity modulation solution (3.8) with constant Riemann invariants

$$
r_{1}=u^{d}\left(1-\alpha u^{d}\right)<0, \quad r_{3}=0,
$$

so that expressions (4.7) for the parameters $u_{k}$ (Region 1 ) reduce to

$$
\begin{aligned}
& u_{1}\left(r_{2}\right)=\frac{1}{2 \alpha}\left(1+2 \alpha u^{d}-\sqrt{1-4 \alpha r_{2}}\right), \\
& u_{2}\left(r_{2}\right)=\frac{1}{2 \alpha}\left(-1+2 \alpha u^{d}+\sqrt{1-4 \alpha r_{2}}\right), \\
& u_{1}\left(r_{2}\right)=\frac{1}{2 \alpha}\left(1-2 \alpha u^{d}-\sqrt{1-4 \alpha r_{2}}\right), \\
& u_{1}\left(r_{2}\right)=\frac{1}{2 \alpha}\left(3-2 \alpha u^{d}+\sqrt{1-4 \alpha r_{2}}\right),
\end{aligned}
$$

and their plots are shown in Fig. 13a. The corresponding modulated solution for the downstream undular bore is shown in Fig. 13b. The speed of the soliton edge is

$$
s_{d}^{-}=\Delta-2 u^{d}\left(1-\alpha u^{d}\right)
$$

and of the linear edge

$$
s_{d}^{+}=\Delta-12 u^{d}\left(1-\alpha u^{d}\right) .
$$

The downstream bore is attached to the topography if $s_{d}^{-}<0$. Similar to the upstream bore, the condition $s_{d}^{-}=0$ yields the 'critical' value of $u^{d}$, which, after the substitution in (2.25) gives the expression of the curve $\Delta^{d}$ separating the regimes corresponding to the attached and detached downstream undular bore

$$
\Delta^{d}: G_{m}(\Delta)=\frac{1}{2 \alpha^{2}}\left\{\frac{1}{2}(1-\sqrt{1-2 \alpha \Delta})^{2}-1+\alpha \Delta+\left(1-\frac{2 \alpha \Delta}{3}\right)^{3 / 2}\right\} .
$$




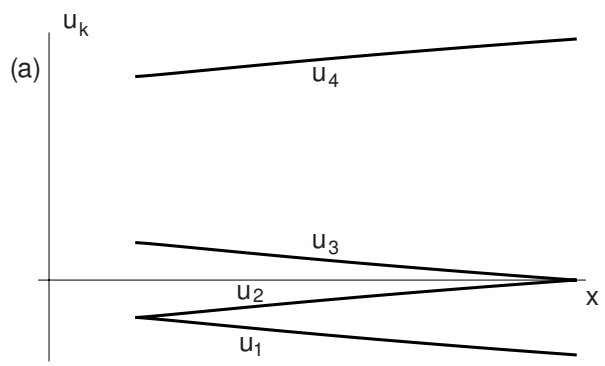

A. M. Kamchatnov et al.

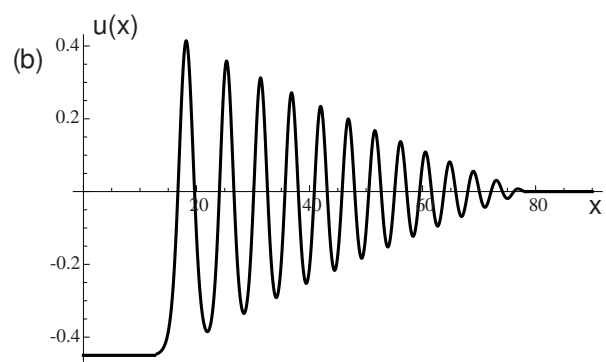

FIGURE 13. Riemann problem solution to the unforced Gardner equation (1.1) with $\alpha>0$ and the initial step parameters $u^{-}=u^{d}<0, u^{+}=0$ (Region 1 in Fig. 7) corresponding to the generation of normal cnoidal undular bore downstream the obstacle. (a) Typical behaviour of the modulation parameters $u_{k}$ on $x$ for some fixed value of time $t>0$. The point with $u_{1}=u_{2}=u^{d}$ corresponds to the soliton edge of the bore; the point with $u_{2}=u_{3}=0$ corresponds to the zero-amplitude (linear) edge. (b) Plot of the analytical (modulation theory) solution to the unforced Gardner equation (1.1) with $\alpha=1, \Delta=0$ and the step-like initial condition (2.31) with $u^{-}=-0.45, u^{+}=0.0$ at the time $t=10$

For small $\alpha|\Delta| \ll 1$ equation (4.21) is approximated by the series expansion (cf. analogous expansion (4.11) for the upstream bore)

$$
G_{m} \alpha^{2} \cong \frac{1}{3}(\alpha \Delta)^{2}+\frac{7}{27}(\alpha \Delta)^{3}
$$

Again, one should take the branch of the inverse function to (4.21) which is located within the region $\Delta_{-}<\Delta<\Delta_{1}$. The curve (4.21) is shown in Fig. 10 (see section 4.1).

One should note that, unlike the partial bores in the upstream resolution, the modulus $m$ in the partial downstream bore ranges in some interval $0<m<m^{*}$, where $m^{*} \leq 1$ so that the partial undular bore downstream generally represents an extended "rear" part of the bore rather than the solitary wavetrain. This type of modulated solutions arises in the KdV initial boundary problem in the negative quarter plane and was studied in Marchant \& Smyth (2002). Since the Gardner modulation theory for the considered region of parameters is equivalent that for the $\mathrm{KdV}$ case we do not consider the details of the downstream partial bores here.

We remark that both curves (4.22) and (4.11) agree to leading order in $|\alpha \Delta| \ll 1$ with the equation of the (single) curve separating the regimes of the attached and detached upstream/downstream bores in the forced KdV equation theory Grimshaw \& Smyth (1986), Smyth (1987). Note that in the forced KdV case, one of the bores is necessarily attached to the obstacle. Contrastingly, in the forced Gardner equation case, there is the region $\Delta^{d}<\Delta<\Delta^{u}$ corresponding to the generation of two detached, fully realised undular bores. A similar configuration with two complete bores occurs in fully nonlinear transcritical single-layer shallow-water flows described by the forced Su-Gardner equation El et al. (2009).

\subsection{Numerical simulations}

The previous study Grimshaw et al. (2002) showed an excellent agreement of the transcritical hydraulic solution to the forced Gardner equation (1.1) with the results of full numerical simulations for a broad range of the input parameters. On the other hand, in Kamchatnov et al. (2012) a detailed comparison of the analytical unsteady modulation solutions for the step resolution problem for the unforced Gardner equation with the numerical solutions of the same problem was carried out. The quantitative comparison 

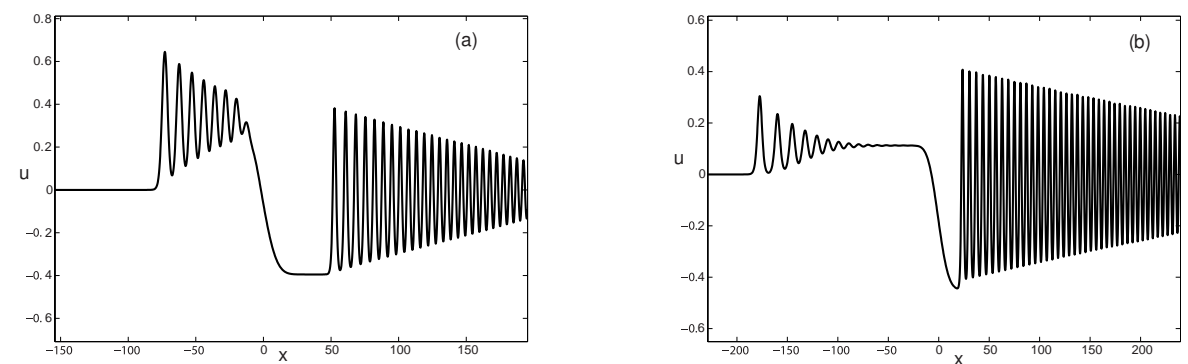

FiguRE 14. Numerical solutions of the forced Gardner equation (1.1) with $\alpha=1$ for different parts of the region $\Delta_{-}<\Delta<\Delta_{1}$ in the parametric flow-regime diagram in Fig. 4a (see also Fig. 10): (a) $\Delta_{-}<\Delta<\Delta^{d},\left(\Delta=-0.44, G_{m}=0.43, t=55\right)$ : the upstream bore is attached, the downstream bore is detached; (b) $\Delta^{u}<\Delta<\Delta_{1},\left(\Delta=-1.34, G_{m}=0.32, t=55\right)$ : the downstream bore is attached, the upstream bore is detached. In both cases the characteristic width of the topography forcing (1.3) was taken $l=10$.

was made for all regions in the classifications presented in Figs. 7 and 8 and a very good agreement with numerics was demonstrated.

Here we mostly concentrate on the verification of the qualitative predictions of our classification of the upstream and downstream unsteady wave patterns detailed in the two previous subsections. As is usually the case with the dispersive transcritical flow problems, the quantitative agreement is very good in the near-critical regime $(\Delta \approx 0)$ and is getting worse when one moves towards the boundaries of the transcritical region of parameters (see e.g. El et al. (2009) or Leszczyszyn et al. (2009)). This is due to much longer times of the establishment of the hydraulic solution far from criticality resulting in a formal violation of the applicability conditions for the Grimshaw \& Smyth (1986) construction.

To verify the predictions of our analysis in Sections 4.1 and 4.2 we have performed three series of simulations using the input parameters $\alpha, G_{m}$ and $\Delta$ from the three distinct subregions of the region $\Delta_{-}<\Delta<\Delta_{+}^{\prime}$ in the parametric flow-regime diagram in Fig. 4a. In our numerical simulations of the forced Gardner equation (1.1) the standard pseudospectral method was used for treatment of the space dependence together with the 4th order Runge-Kutta procedure for time stepping (see e.g. Trefethen (2000)). The typical numerical results from all three series are presented in Figs. 14-17. As one can see, the numerical simulations completely agree with the predictions of our classification.

\section{Upstream and downstream closure, $\alpha<0$.}

An analysis of the dispersive resolution of the upstream and downstream hydraulic jumps in the transcritical Gardner equation with $\alpha<0$ is in many respects analogous to that performed in sections 4.1 and 4.2 for the case $\alpha>0$. However, due to some distinctive features of the periodic travelling wave solutions of the Gardner equation with $\alpha<0$ resulting in non-strict hyperbolicity of the modulation system, the structure of the downstream and upstream waves generated in the transcritical Gardner flows can drastically differ from that in the dispersive resolution patterns described in the previous section. The special role in the dispersive step resolutions in the Gardner equation (1.1) with $\alpha<0$ is played (for a certain range of the initial step parameters) by the socalled trigonometric undular bores, which were first described in Marchant (2008) for the focusing $\mathrm{mKdV}$ equation, and which are, in some sense, the counterparts of the solibores 


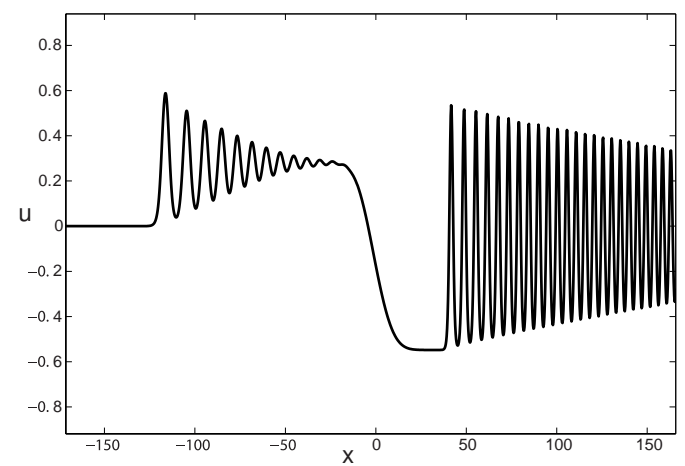

FIgURE 15. Numerical solution of the forced Gardner equation (1.1) with $\alpha=1$ for the region $\Delta^{d}<\Delta<\Delta^{u}$ of the input parameters (see Figs. 4a and 10): both undular bores are detached from the obstacle. The parameters in the numerical solutions are $\Delta=-1.25, G_{m}=0.66, t=95$. The characteristic width of the topography forcing (1.3) was taken $l=10$.

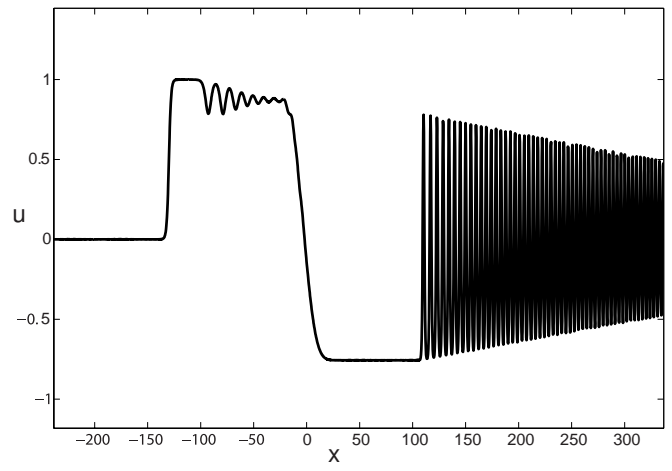

FIGURE 16. Numerical solution of the forced Gardner equation (1.1) with $\alpha=1$ for the region $\Delta_{1}<\Delta<\Delta_{2}$ of the input parameters (see Fig. 4a and 10). Upstream is an attached reversed undular bore connected to the equilibrium flow by a solibore (cf. the relevant analytical solution plot in Fig. 11b); downstream a detached normal undular bore is generated. The parameters in the numerical solution are: $\alpha=1, \Delta=-1, G_{m}=1.9, l=10, t=60$.

generated in the 'lock-exchange' flows of the Gardner equation with $\alpha>0$. The detailed analysis of the Gardner equation trigonometric bores can be found in Kamchatnov et al. (2012).

\subsection{Upstream solution}

Similar to the case $\alpha>0$, in order to describe the upstream wave pattern we need to solve the unforced Gardner equation (1.1) with initial conditions (2.31) where $u^{-}=0$ and $u^{+}=u^{u}>0$. The dependence of $u^{u}$ on the definitive parameters $\Delta, G_{m}$ and $\alpha$ is given by the same equations $(2.25),(2.26)$. The value of $u^{u}$ within the region of existence of the localised transcritical hydraulic solution (see Fig. 4b) ranges now from $\left(u^{u}\right)_{\min }=0$ at the upper boundary $\Delta_{+}$to some $\left(u^{u}\right)_{\max }$ at the lower boundary $\Delta_{-}^{\prime}$. The value $\left(u^{u}\right)_{\max }$ is found as the smaller positive root of equation (2.25) with $G_{m}(\Delta)$ defined by $(2.29)$.

An inspection of the classification presented in Fig. 8 shows that within this range of $u^{+}=u^{u}$ (at fixed $u^{-}=0$ ) one can possibly have only one type of the upstream 


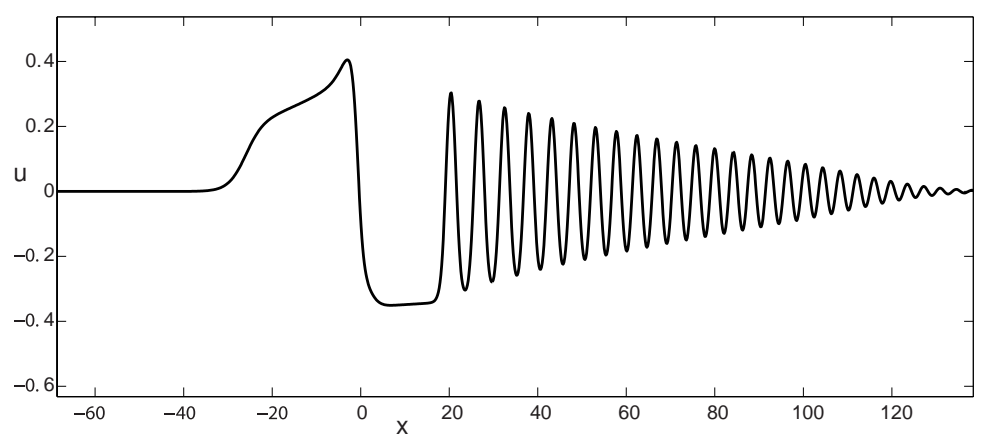

FIGURE 17. Numerical solution of the forced Gardner equation (1.1) with $\alpha=1$ for the region $\Delta_{2}<\Delta<\Delta_{+}^{\prime}$ of the input parameters (see Fig. 4a). Upstream resolution pattern: a combination of a rarefaction wave and a solibore (cf. the relevant analytical solution plot in Fig. 12); downstream - a detached undular bore. The parameters in the numerical solution are: $\alpha=4.8$, $\Delta=-1, G_{m}=0.33, l=4, t=20$.

resolution - the one corresponding to Region 4 . The relevant structure is the KdV type "normal" cnoidal undular bore with a bright soliton at the leading edge. Locally, this bore is described by the following periodic solution of the basic ODE (3.1) (Kamchatnov et al. (2012)),

$$
u=u_{3}+\frac{\left(u_{4}-u_{3}\right) \operatorname{cn}^{2}\left(\theta, m_{2}\right)}{1+\frac{u_{4}-u_{3}}{u_{3}-u_{1}} \operatorname{sn}^{2}\left(\theta, m_{2}\right)},
$$

where

$$
\begin{gathered}
\theta=\sqrt{|\alpha|\left(u_{3}-u_{1}\right)\left(u_{4}-u_{2}\right)}\left(x-x_{0}-V t\right) / 2, \\
m=\frac{\left(u_{4}-u_{3}\right)\left(u_{2}-u_{1}\right)}{\left(u_{4}-u_{2}\right)\left(u_{3}-u_{1}\right)}
\end{gathered}
$$

and $V$ is given by (4.4).

For Region 4, the expressions for the parameters $u_{j}$ of the periodic solution (5.1) in terms of the Riemann invariants $r_{1}, r_{2}, r_{3}$ in the 'universal' modulation solution (3.7), (3.8) are given by the following inverse formulae of (3.5) (see Kamchatnov et al. (2012)),

$$
\begin{aligned}
& u_{1}=\frac{1}{2 \alpha}\left(1+\sqrt{1-4 \alpha r_{1}}+\sqrt{1-4 \alpha r_{2}}+\sqrt{1-4 \alpha r_{3}}\right), \\
& u_{2}=\frac{1}{2 \alpha}\left(1-\sqrt{1-4 \alpha r_{1}}-\sqrt{1-4 \alpha r_{2}}+\sqrt{1-4 \alpha r_{3}}\right), \\
& u_{3}=\frac{1}{2 \alpha}\left(1-\sqrt{1-4 \alpha r_{1}}+\sqrt{1-4 \alpha r_{2}}-\sqrt{1-4 \alpha r_{3}}\right), \\
& u_{4}=\frac{1}{2 \alpha}\left(1+\sqrt{1-4 \alpha r_{1}}-\sqrt{1-4 \alpha r_{2}}-\sqrt{1-4 \alpha r_{3}}\right) .
\end{aligned}
$$

The constant Riemann invariants (3.7) are

$$
r_{1}=0, \quad r_{3}=u^{u}\left(1-\alpha u^{u}\right),
$$



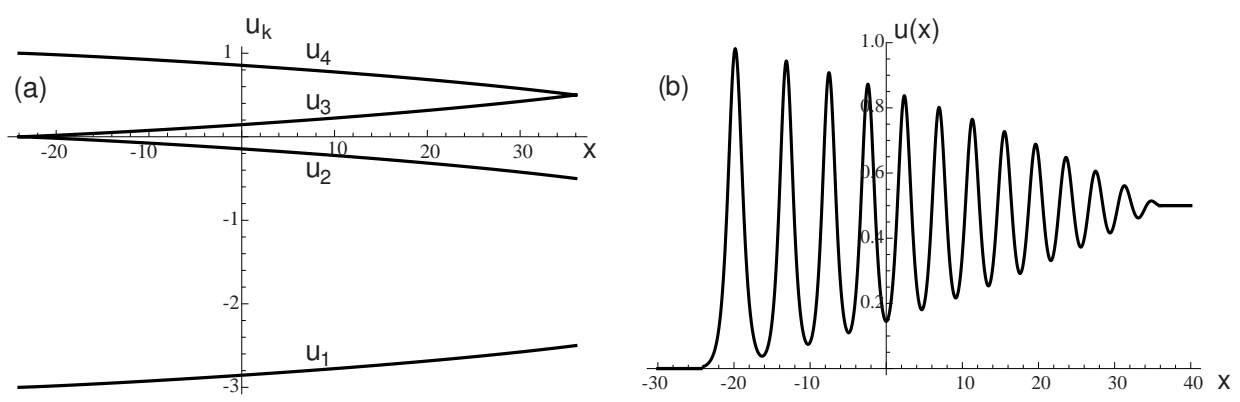

FIGURE 18. Riemann problem solution to the unforced Gardner equation (1.1) with $\alpha<0$ and the initial step parameters $u^{-}=0, u^{+}=u^{u}>0$ corresponding to the upstream resolution via normal undular bore (Region 4 in Fig 8). (a) Typical behaviour of the modulation parameters $u_{k}$ in the bore. At the soliton edge $u_{3}=u_{2}=0$, at the linear zero-amplitude edge $u_{3}=u_{4}$; (b) The plot of the analytical (modulation theory) solution to the unforced Gardner equation (1.1) with $\alpha=-1, \Delta=0$ and the step-like initial condition (2.31) with $u^{-}=0, u^{+}=0.5$ at the time $t=8$. Only the upstream part, $x<0$, of the bore is realised in the transcritical flow.

so that expressions (5.4) assume the form

$$
\begin{aligned}
& u_{1}\left(r_{2}\right)=\frac{1}{2 \alpha}\left(3-2 \alpha u^{u}+\sqrt{1-4 \alpha r_{2}}\right) \\
& u_{2}\left(r_{2}\right)=\frac{1}{2 \alpha}\left(1-2 \alpha u^{u}-\sqrt{1-4 \alpha r_{2}}\right) \\
& u_{3}\left(r_{2}\right)=\frac{1}{2 \alpha}\left(-1+2 \alpha u^{u}+\sqrt{1-4 \alpha r_{2}}\right) \\
& u_{4}\left(r_{2}\right)=\frac{1}{2 \alpha}\left(1+2 \alpha u^{u}-\sqrt{1-4 \alpha r_{2}}\right) .
\end{aligned}
$$

The dependence of $r_{2}$ on $x, t$ is determined implicitly by the similarity modulation solution Eq. (3.8) so the modulations $u_{j}(x, t)(5.6)$ are completely defined. When $r_{2}$ varies between $r_{1}$ and $r_{3}$ the parameters $u_{j}$ vary along the curves shown in Fig. 18a. The corresponding plot of the undular bore constructed by substituting the modulation solution (5.6), (3.8) in the travelling wave (5.1) is shown in Fig. 18b. The speeds of the soliton and linear edges of the upstream undular bore are

$$
s_{u}^{-}=\Delta-4 u^{u}\left(1-\alpha u^{u}\right) \text { and } s_{u}^{+}=\Delta+6 u^{u}\left(1-\alpha u^{u}\right)
$$

respectively. Since $u^{u}>0, \alpha<0$ and $\Delta|\alpha|>-1$ (see Fig. 4b) it is not difficult to show that $s_{u}^{+}>0$ for all values of the input parameters $G_{m}, \Delta$ involved. Thus, for $\alpha<0$ the upstream undular bore can only be realised partially. As was mentioned earlier, the partial undular bore can often be approximated by a uniform train of solitary waves (see Grimshaw \& Smyth (1986), Smyth (1987) for the similar issue for the forced KdV equation). This approximation is expected to work particularly well for large values of the normalised topography amplitude $G_{m} \alpha^{2}$ leading to the "cutoff" modulus $m_{0}$ values sufficiently close to unity.

\subsection{Downstream solution}

To describe the downstream wave pattern we need to solve the unforced Gardner equation (1.1) with initial conditions (2.31) where $u^{-}=u^{d}<0$ and $u^{+}=0$. The dependence of $u^{d}$ on the definitive parameters $\Delta, G_{m}$ is given by (2.25), (2.26). The value of $u^{d}$ within the region of the existence of the localised transcritical hydraulic solution (see Fig. 4b) ranges from $u^{d}=0$ at the upper boundary $\Delta_{+}$to some $\left(u^{d}\right)_{\min }<0$ at the lower boundary $\Delta_{+}^{\prime}$. 

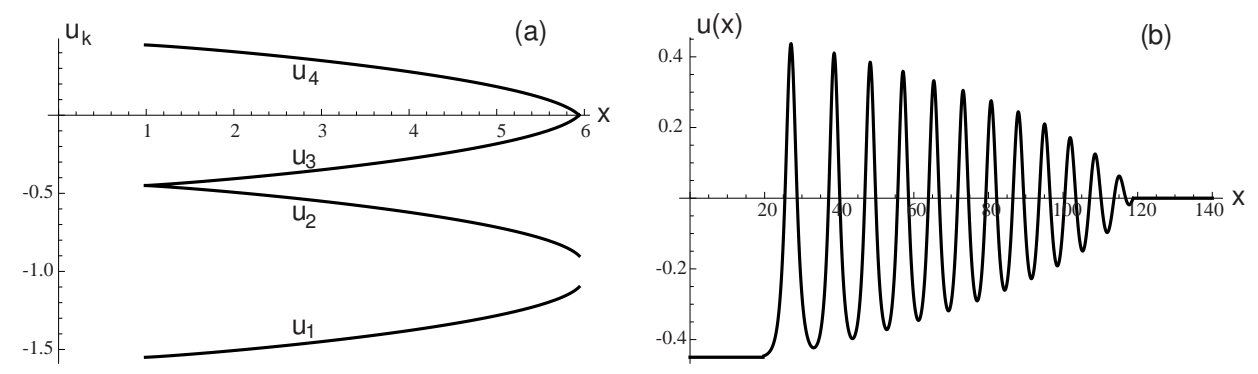

Figure 19. Riemann problem solution to the unforced Gardner equation (1.1) with $\alpha<0$ and the initial step parameters $u^{+}=0, u^{-}=u^{d}$, where $1 /(2 \alpha)<u^{d}<0$, corresponding to the region $\Delta_{1}<\Delta<\Delta_{+}$in the transcritical flow-regime diagram in Fig. 4b (Region 4 in Fig. 8). (a) Typical behaviour of the modulation parameters $u_{k}$ in the normal undular bore generated downstream the obstacle in the transcritical flow. At the soliton edge $u_{3}=u_{2}=u^{d}$; at the linear edge $u_{3}=u_{4}=0$. (b) Plot of the analytical (modulation theory) solution to the Gardner equation with $\alpha=-1, \Delta=0$ and the step-like initial condition (2.31) with $u^{-}=-0.45$, $u^{+}=0.0$ at the time $t=40$.

The value $\left(u^{d}\right)_{\min }$ is found as the negative root of equation (2.25) (see Fig. 3b) with $G_{m}(\Delta)$ defined by $(2.29)$.

An inspection of the classification presented in Fig. 8 shows that, within this range of $u^{-}=u^{d}<0$ (at fixed $u^{-}=0$ ) one can possibly have three different types of behaviours corresponding to Regions 4, 3 and 2 respectively. The corresponding solutions are outlined below.

(a) $\Delta_{1}<\Delta<\Delta_{+}$This region of the parametric diagram in Fig. 4b corresponds to the initial steps $(2.31)$ with $1 /(2 \alpha)<u^{-}<0, u^{+}=0$, and, therefore, to Region 4 in the classification map in Fig. 8. Thus we have the classical KdV type "bright" cnoidal bore generated downstream the obstacle. Similar to the upstream undular bore described in the previous subsection, the oscillatory structure of the undular bore for the considered range of the input parameters is described by the travelling wave solution (5.1) with the relations (5.4) between the modulation parameters $u_{j}, j=1,2,3,4$ and the Riemann invariants $r_{1}, r_{2}, r_{3}$. The constant Riemann invariants in the Gurevich-Pitaevskii solution (3.7), (3.8) now are

$$
r_{1}=u^{d}\left(1-\alpha u^{d}\right), \quad r_{3}=0
$$

which leads to the following set of expressions for $u_{j}$ 's (cf. (5.6))

$$
\begin{aligned}
& u_{1}\left(r_{2}\right)=\frac{1}{2 \alpha}\left(3-2 \alpha u^{d}+\sqrt{1-4 \alpha r_{2}}\right), \\
& u_{2}\left(r_{2}\right)=\frac{1}{2 \alpha}\left(1+2 \alpha u^{d}-\sqrt{1-4 \alpha r_{2}}\right), \\
& u_{3}\left(r_{2}\right)=\frac{1}{2 \alpha}\left(-1+2 \alpha u^{d}+\sqrt{1-4 \alpha r_{2}}\right), \\
& u_{4}\left(r_{2}\right)=\frac{1}{2 \alpha}\left(1-2 \alpha u^{d}-\sqrt{1-4 \alpha r_{2}}\right) .
\end{aligned}
$$

The speeds of the downstream undular bore soliton and linear edges are

$$
s_{d}^{-}=\Delta-2 u^{d}\left(1-\alpha u^{d}\right) \text { and } s_{d}^{+}=\Delta-12 u^{d}\left(1-\alpha u^{d}\right)
$$

respectively. The soliton amplitude is $a=-2 u^{d}$. The condition $s_{d}^{-}=0$ corresponds to the bore whose soliton edge is attached to the obstacle. Expressing $u^{d}$ from this condition and substituting it into equation (2.25) we obtain the same equation (4.21) obtained for the line $\Delta^{d}$ separating the regimes of the attached (partial) and detached (fully 
realised) downstream undular bore for the case $\alpha>0$. The only difference is that now, upon inversion of (4.21), one chooses the upper branch of the function $\Delta=\Delta^{d}\left(G_{m}\right)$. As a result, the partial downstream bore can only be realised in a very small sector of parameters with $\Delta<0$ in the flow-regime diagram in Fig. 4b. One should also note that, unlike partial bores in the upstream resolution for $\alpha>0$ (see Section 4) the modulus $m$ in the partial downstream bore ranges in some interval $0<m<m_{0}$, where $m_{0} \leq 1$. This type of modulated solution for the KdV equation was studied in Marchant \& Smyth (2002). Since the Gardner modulation theory for the considered region of parameters is equivalent that for the $\mathrm{KdV}$ case, we do not consider the details of the downstream partial bores here.

(b) $\Delta_{2}<\Delta<\Delta_{1}$. This region of the parametric diagram in Fig. 4b corresponds to the initial steps $(2.31)$ with $1 / \alpha<u^{-}<1 /(2 \alpha), u^{+}=0$, and, therefore, to Region 3 in the classification map in Fig. 8.

The wave structure generated downstream is now a composite undular bore, which consists of two parts. The front (left) part is described by the cnoidal solution (5.1) in which the modulus $m$ varies from $m=1$ at the leading edge to $m=0$ at some point $x^{*}$, which, however is not the bore's trailing edge as the amplitude $a$ does not vanish at this point. The structure then continues via the modulated nonlinear trigonometric solution of the Gardner equation, in which $u_{2}=u_{1}$ (i.e. $m=0$ - see (5.3)) but $a=u_{4}-u_{3} \neq 0$. This solution has the form

$$
u=u_{3}+\frac{\left(u_{4}-u_{3}\right) \cos ^{2} \theta}{1+\frac{u_{4}-u_{3}}{u_{3}-u_{1}} \sin ^{2} \theta},
$$

where

$$
\begin{aligned}
& \theta=\sqrt{|\alpha|\left(u_{3}-u_{1}\right)\left(u_{4}-u_{1}\right)}\left(x-x_{0}-V t\right) / 2, \\
& V=\Delta-4 u_{1}-\alpha\left(u_{3} u_{4}-3 u_{1}^{2}\right) .
\end{aligned}
$$

The modulated trigonometric solution (5.11) describes the rear (right) part of the downstream composite undular bore through the trailing edge where $a \rightarrow 0$. The details of the analytical description of the 'trigonometric' undular bores in the Gardner equation can be found in Kamchatnov et al. (2012); here we apply the relevant solutions from Kamchatnov et al. (2012) to the particular configuration of the downstream resolution in the transcritical flow.

The modulation in the cnoidal part of the bore is described by the same expressions (5.9), where $r_{2}(x, t)$ varies according to the Gurevich-Pitaevskii solution (3.7), (3.8) with the same as in (5.8) constant Riemann invariants

$$
r_{1}=u^{d}\left(1-\alpha u^{d}\right), \quad r_{3}=0 .
$$

The difference between the solution in the present case $1 / \alpha<u^{d}<1 /(2 \alpha)$ and the previous case (a) $1 /(2 \alpha)<u^{-}<0, u^{+}=0$ is that now one has to choose in (5.9) the other branch of $\sqrt{1-4 r_{2}}$. As a result, the amplitude $a=u_{4}-u_{3}$ does not vanish at the edge with $r_{1}=r_{2}(m=0)$. Indeed, one can readily see that $a=8 \alpha u^{d}>0$ at this point $x=x^{*}$, which coincides with position of the trailing edge in the Gurevich-Pitaevskii solution (3.8). Then, to get the required matching with $u=0$ downstream, one has to continue the solution beyond $x=x^{*}$, via the modulated nonlinear trigonometric wave (5.11) along which $\left.v_{2}\right|_{m=0}=x / t$ that is

$$
r_{1}=r_{2}=r=\frac{1}{12}\left(\Delta-\frac{x}{t}\right) .
$$



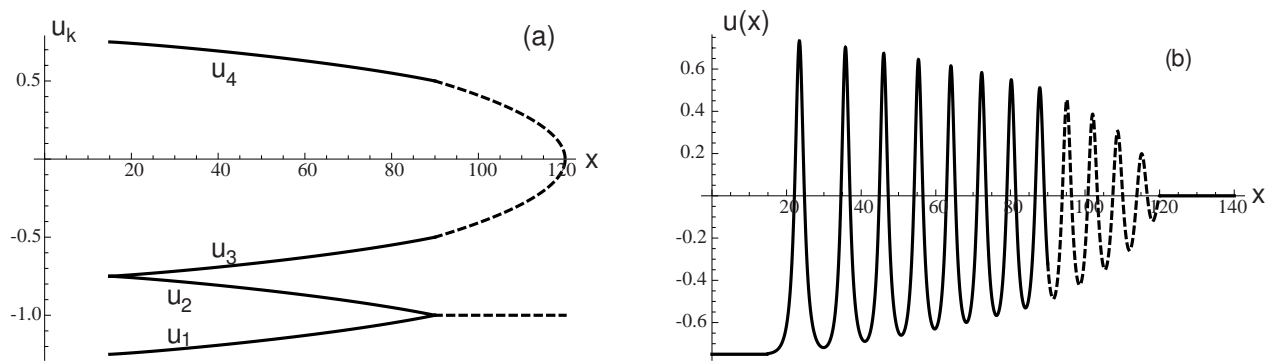

Figure 20. Riemann problem solution to the unforced Gardner equation (1.1) with $\alpha<0$ and $u^{+}=0$ and $u^{-}=u^{d}$, where $1 / \alpha<u^{d}<1 /(2 \alpha)$ corresponding to the downstream resolution via a composite cnoidal-trigonometric undular bore in the transcritical flow with the parameters $\Delta, G_{m}$ located in the region $\Delta_{2}<\Delta<\Delta_{1}$ of the parametric flow-regime diagram in Fig. $4 \mathrm{~b}$ and Region 3 in the solution classification in Fig. 8. (a) Typical behaviour of the modulation parameters $u_{k}$ in the cnoidal-trigonometric undular bore. The point with $u_{2}=u_{3}=u^{d}$ corresponds to the leading, soliton edge; the point with $u_{3}=u_{4}=0$ corresponds to the linear trailing edge. The boundary between the cnoidal and trigonometric part of the bore is located at the point where $u_{2}=u_{1}$. (b) Plot of the analytical (modulation theory) solution of the Gardner equation with $\alpha=-1$ and $\Delta=0$ and step-like initial conditions (2.31) with $u^{-}=-0.75, u^{+}=0.0$ at the time $t=40$.

This solution extends up to $x=x^{+}=(\Delta-3 / \alpha) t$ where $u_{3}=u_{4}(a=0)$. The variations of the modulation parameters $u_{k}$ in the trigonometric part of the bore are given by

$$
u_{1}=u_{2}=\frac{1}{\alpha}, \quad u_{3}=\frac{1}{\alpha} \sqrt{1-\frac{\alpha}{3}\left(\Delta-\frac{x}{t}\right)}, \quad u_{4}=-\frac{1}{\alpha} \sqrt{1-\frac{\alpha}{3}\left(\Delta-\frac{x}{t}\right)} .
$$

The typical variations of $u_{k}$ as functions of $x$ in the composite cnoidal-trigonometric bore are shown in Fig. 20a and the corresponding oscillatory structure is shown in Fig. 20b. It is worth noticing that the presence of the trigonometric part of the bore leads to a slower transition to the linear wave packet at the small amplitude edge compared with a pure cnoidal bore. The linear edge of the composite bore propagates with the velocity

$$
s^{+}=\Delta-\frac{3}{\alpha} .
$$

The boundary $x=x^{*}$ between the cnoidal and the trigonometric parts propagates with the velocity

$$
s^{*}=\Delta-12 u^{-}\left(1-\alpha u^{-}\right)
$$

coinciding with the velocity of the trailing edge in the Gurevich-Pitaevskii solution (the second formula (5.10)). The soliton edge propagates with the velocity $s^{-}$given by the first formula (5.10) and the lead soliton amplitude is

c) $\Delta_{-}^{\prime}<\Delta<\Delta_{2}$. This region of the parametric diagram in Fig. 4b corresponds to the initial steps (2.31) with $u_{\min }^{d}<u^{-}<1 / \alpha, u^{+}=0$, where $u_{\min }^{d}$ is the smallest root of the equation (2.25) with $\Delta=\Delta_{-}^{\prime}$ defined by (2.29). This range of the initial steps corresponds to Region 2 in the classification chart in Fig. 8. Note that in this region $\Delta>0$. The wave structure generated downstream is now a combination of a reversed rarefaction wave and a 'normal' trigonometric bore. This structure is a counterpart of the combined solibore-rarefaction wave solution in the $\alpha>0$ case. The trigonometric bore connects the equilibrium state $u=0$ at the right edge $x=s^{+} t$ and the 'conjugate' state $u=1 / \alpha<0$ at the left edge $x=s^{-} t$ and is described by the periodic solution 


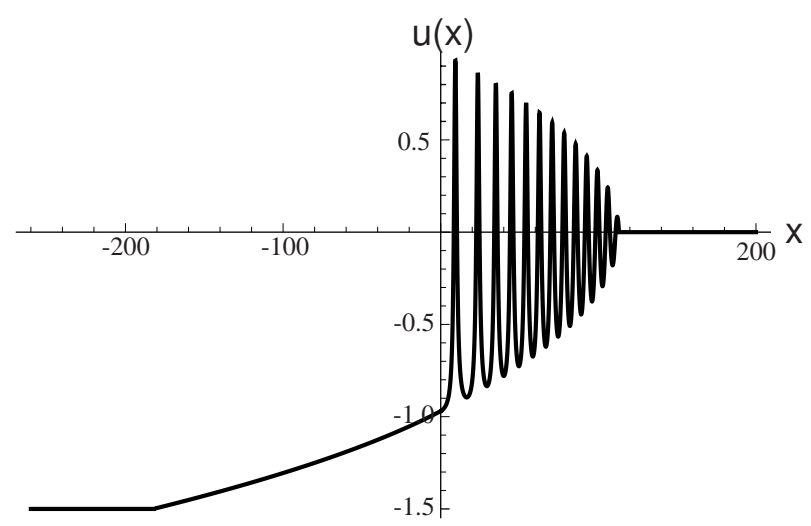

FiguRE 21. Plot of the analytical (modulation theory) solution of the unforced Gardner equation (1.1) with $\alpha<0$ and the initial parameters $u^{+}=0, u^{-}=u^{d}$, where $u_{\min }^{d}<u^{d}<1 / \alpha$, corresponding to the downstream resolution of the transcritical flow with parameters $\Delta, G_{m}$ located in the region $\Delta_{-}^{\prime}<\Delta<\Delta_{2}$ of the parametric flow-regime diagram in Fig. $4 \mathrm{~b}$. The resolution pattern consists of the reversed rarefaction wave and normal trigonometric bore joined together at the point with $u=1 / \alpha$ (see Region 4 in the classification chart in Fig. 8 ). The plot parameters are: $\alpha=-1, \Delta=0, u^{-}=-1.5, u^{+}=0, t=40$.

(5.11), (5.12) modulated according to (5.15). The edge speeds are

$$
s^{-}=\Delta, \quad s^{+}=\Delta-\frac{3}{\alpha} .
$$

Since in the region considered we have $\Delta>0$, the trigonometric bore is fully realised (cf. case (b) above). At the leading edge $x=s^{-} t$ the bore has an algebraic soliton, described by the limit of the trigonometric solution (5.11) as $u_{3} \rightarrow u_{1}$

$$
\begin{aligned}
& u=u_{1}+\frac{u_{4}-u_{1}}{1+|\alpha|\left(u_{4}-u_{1}\right)^{2}(x-V t)^{2} / 4}, \\
& V=\Delta-6 u_{1}\left(1-\alpha u_{1}\right) .
\end{aligned}
$$

Using relationship (3.3) and the fact that the pedestal of the algebraic soliton $u=u_{1}=$ $1 / \alpha$ we reduce $(5.19)$ to the form

$$
u=\frac{1}{\alpha}\left(1-\frac{2}{1+\frac{4}{|\alpha|}(x-\Delta t)}\right) .
$$

The algebraic soliton (5.20) is joined to the downstream transcritical state $u=u^{d}$ right behind the obstacle by the reversed rarefaction wave, which is asymptotically described by the following similarity solution of the dispersionless Gardner equation (1.1) (cf. (4.15))

$$
\begin{aligned}
& u=u^{d}, \quad \text { for } \quad x<s^{l} t, \\
& u=\frac{1}{2 \alpha}\left(1+\sqrt{1+\frac{2 \alpha}{3}\left(\frac{x}{t}-\Delta\right)}\right) \text { for } s^{l} t<x<s^{r} t, \\
& u=\frac{1}{\alpha} \text { for } \quad x>s^{r} t .
\end{aligned}
$$

The speeds of the rarefaction wave edges are

$$
s^{l}=\Delta-6 u^{d}\left(1-\alpha u^{d}\right), \quad s^{r}=\Delta,
$$

so the speed of the right edge of the rarefaction wave coincides with the speed of the 


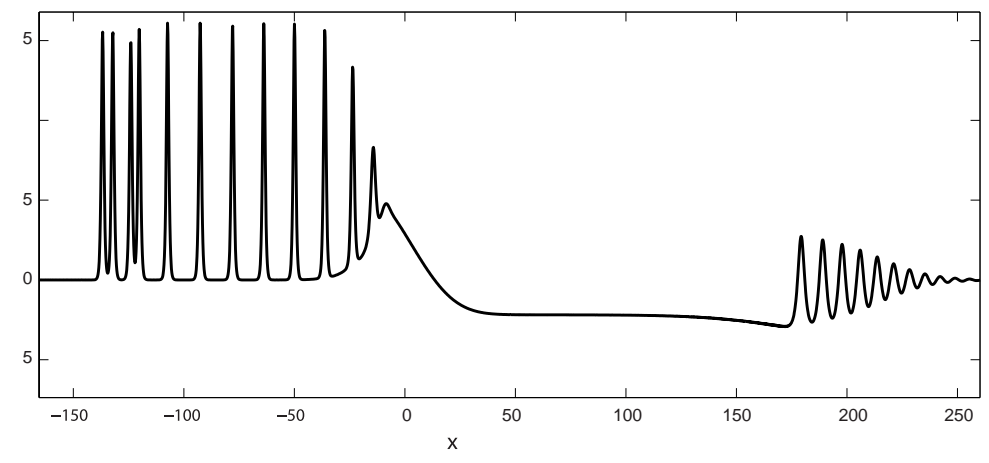

FIGURE 22. Numerical solutions of the forced Gardner equation (1.1) with $\alpha=-1$ for $\Delta_{1}<\Delta<\Delta_{+}$(see the parametric flow-regime diagram in Fig. 4b). The generated waves are: the partial cnoidal undular bore (solitary wave train) upstream and fully realised cnoidal bore downstream. The plot parameters are: $\Delta=1.49, G_{m}=0.44, l=20, t=85$.

algebraic soliton in the trigonometric bore implying that there is no additional constant states between the two structures. Also it is not difficult to check that $0<s^{l}<s^{r}$ for the considered range of the input parameters so the rarefaction wave never gets attached to the obstacle. The above-described downstream composite solution is illustrated in Fig. 21.

\subsection{Numerical simulations}

In this section we present numerical solutions of the forced Gardner equation (1.1) with $\alpha<0$ and the forcing term defined by formula (1.3). Similar to the case $\alpha>0$ (see the numerical section 4.3), to verify the predictions of our analysis in Sections 5.1 and 5.2 we have performed three series of simulations using the input parameters $\alpha, G_{m}$ and $\Delta$ from the three distinct subregions of the region $\Delta_{-}^{\prime}<\Delta<\Delta^{+}$in the parametric flow-regime diagram in Fig. 4b. In Figs. 22-24 we present some of the typical upstream and downstream configurations illustrating our classification in the previous subsections. One can see that in all three plots the upstream bore is realised partially and attached to the obstacle as predicted by our analysis. Some irregularities in the structure of the leading part of the partial bore (or rather solitary wave train) in Figs. 22 and 24 is explained by the 'history' of its generation. Namely, at the initial stage of the establishment of the steady transcritical hydraulic regime over the obstacle, a series of smaller solitary waves are generated. These propagate downstream but eventually are overtaken by larger solitary waves continuously generated at the obstacle's location after the hydraulic transcritical transition has settled. The relative width of the irregular part of the bore decreases with time so for sufficiently large $t$ can be safely neglected (see the longtime simulation in Fig. 23). In Fig. 23 one can also clearly see the "wine-glass" shape of the envelope of the downstream bore, which is the characteristic feature of the composite, cnoidal-trigonometric bore described in Section 5.2 and illustrated in the analytical plot in Fig. 20b. Finally, in Fig. 24 one can observe the downstream pattern consisting of an undular bore and a rarefaction wave, which agrees with the predicted theoretical pattern shown in Fig. 21.

\section{Conclusion}

The classification of the transcritical (resonant) flows of a stratified fluid over topography is constructed in the framework of the Gardner (extended KdV) equation (1.1) 


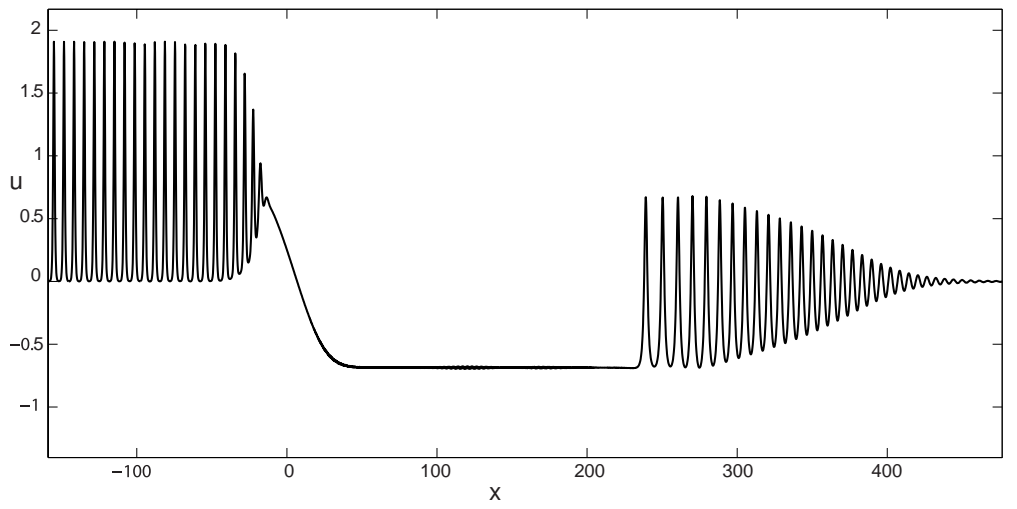

Figure 23. Numerical solution of the forced Gardner equation (1.1) with $\alpha=-1$ for $\Delta_{+}<\Delta<\Delta_{2}$ (see the parametric flow-regime diagram in Fig. 4b). The generated waves are: the partial cnoidal undular bore (solitary wave train) upstream and the composite cnoidal-trigonometric undular bore downstream (cf. the analytical curve in Fig. 20). The plot parameters are: $\Delta=1.89, G_{m}=2.31, l=20, t=85$.

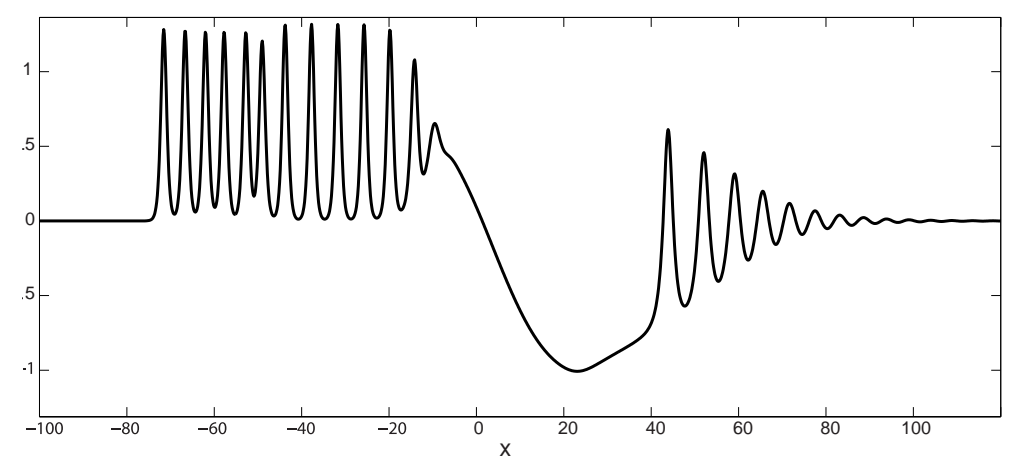

FiguRE 24. Numerical solutions of the forced Gardner equation (1.1) with $\alpha=-1$ for the region $\Delta_{-}^{\prime}<\Delta<\Delta_{2}$ of the parametric flow-regime diagram in Fig. 4b. The generated waves are: the partial cnoidal undular bore (solitary wave train) upstream and the composite pattern consisting of the normal trigonometric undular bore and the reversed rarefaction wave downstream (cf. analytical curve in Fig. 21). The plot parameters are: $\Delta=0.595, G_{m}=1.64, l=10, t=20$.

for a range of flow parameters, when the obstacle supports a steady hydraulic transition from the subcritical flow upstream to the supercritical flow downstream. We consider both possible signs for the cubic nonlinear term $\alpha$ in the Gardner equation corresponding to different fluid density stratification profiles. This problem has been considered in a number of earlier studies using various approaches, but mostly numerically with no full analytical description available.

The main contribution of the present paper is the development of the missing analytical theory of the transcritical Gardner flows in the framework of the Grimshaw \& Smyth (1986) approach, where the asymptotic description of the the flow is constructed using a combination of the hydraulic approximation over the obstacle's location and the modulation description of the unsteady waves generated upstream and downstream. The extension of the GS theory, which was originally developed for the forced KdV equation, to the forced Gardner equation is quite nontrivial and has become possible due to the 
recent development in Kamchatnov et al. (2012) of the full modulation theory for the (unforced) Gardner equation.

We have shown that, along with the regular cnoidal undular bores occurring in the analogous problem for the single-layer flow modeled by the forced KdV equation and its non-integrable extensions (see El et al. (2009)), the forced Gardner equation supports a diverse family of upstream and downstream wave structures which are sharply different from those arising in the forced KdV equation dynamics. These new structures include combinations of solibores and rarefaction waves, "reversed" undular bores and the socalled "trigonometric" undular bores. The classification of the transcritical flow regimes is made in terms of two definitive parameters: the obstacle's height $G_{m}$ and the deviation $\Delta$ of the incident flow velocity from the long wave phase speed. This is done using a combination of the detailed analysis of the hydraulic solution of the forced Gardner equation with the dispersive term neglected and the relevant parts of the Riemann problem solution of the full unforced Gardner equation available from Kamchatnov et al. (2012). The latter work has enabled the analytical description of the unsteady wave structures generated upstream and downstream of the obstacle. The predictions of the constructed analytical theory are confirmed by direct numerical computations of the forced Gardner equation.

Apart from being fundamentally important as the basic analytical model for the weakly nonlinear transcritical flows in stratified fluids, the developed theory should provide a guidance for similar classifications for the more advanced, fully nonlinear models of the transcritical internal flows past topography, for example, the forced Myatta-ChoiCamassa equations for the two-layer flows past topography, which would generalise the theory of the fully nonlinear shallow-water single layer transcritical flows constructed in El et al. (2009).

\section{Acknowledgements}

We acknowledge helpful discussions with Mark Hoefer, Eduardo Khamis and Maxim Pavlov. AMK thanks National Taiwan University and Taida Institute for Mathematical Sciences, where this work was started, for kind hospitality.

\section{REFERENCES}

AKYLAS, T. R. 1984 On the excitation of long nonlinear water waves by a moving pressure distribution. J. Fluid Mech. 141, 455-466.

Apel, J. R., Ostrovsky, L. A., Stepanyants, Y. A. \& Lynch, J. F. 2007 Internal solitons in the ocean and their effect on underwater sound. J. Acoust. Soc. Am. 121, 695-722.

Baines, P. G. 1984 A unified description of two-layer flow over topography. J. Fluid Mechanics 146, $127-167$.

Baines, P. G. 1995 Topographic effects in stratified flows. Cambridge University Press, Cambridge.

Cole, S. L. 1985 Transient waves produced by flow past a bump. Wave Motion 7, 579-587.

El, G. A., Grimshaw, R. H. J. \& Sмyтh, N. F. 2009 Transcritical shallow-water flow past topography: finite-amplitude theory. J. Fluid Mechanics 640, 187-214.

Esler, J. G. \& Pierce, J. D. 2011 Dispersive dam-break and lock-exchange flows in a two-layer fluid. J. Fluid Mechanics 667, 555-585.

Fornberg, B. \& Whitham, G. B. 1978 A numerical and theoretical study of certain nonlinear wave phenomena. Philosophical Transactions of the Royal Society A 289, 373-404.

Grimshaw, R. 2001 Environmental Stratified Flows. Kluwer Academic, Boston.

Grimshaw, R. H. J., Chan, K. H. \& Chow, K. W. 2002 Transcritical flow of a stratified fluid: the forced extended Korteweg - de Vries model. Phys. Fluids 14, 755-774. 
Grimshaw, R. H. J. \& Smyth, N. F. 1986 Resonant flow of a stratified fluid over topography. J. Fluid Mechanics 697, 237-272.

Gurevich, A. V. \& Pitaevskit, L. P. 1974 Nonstationary structure of a collisionless shock wave. Sov. Phys. JETP 38, 291-297.

Helfrich, K. R. \& Melville, W. K. 2006 Long nonlinear internal waves. Ann. Rev. Fluid Mech 38, 395-425.

Holloway, P., Pelinovsky, E. \& Talipova, T. 2001 Internal tide transformation and oceanic internal solitary waves. In Environmental Stratified Flows (ed. R. Grimshaw), pp. 31-60. Boston: Kluwer.

Kakutani, T. \& Yamasaki, N. 1978 Solitary waves on a two-layer fluid. J. Phys. Soc. Japan. 45, 674-679.

Kamchatnov, A. M., Kuo, Y.-H., Lin, T.-C., Horng, T.-L., Gou, S.-C., Clift, R., El, G. A. \& Grimshaw, R. H. J. 2012 Undular bore theory for the Gardner equation. Phys. Rev. E 86, 036605

Kodama, Y., Pierce, V. U. \& Tian, F.-R. 2008 On the Whitham equations for the defocusing complex modified KdV equation. SIAM J. Math. Anal. 41, 26-58.

LEFloch, P. G. 2002 Hyperbolic systems of conservation laws. Birkhauser.

Leszczyszyn, A. M., El, G. A., Gladush, Yu. G. \& Kamchatnov, A. M. 2009 Transcritical flow of a Bose-Einstein condensate through a penetrable barrier. Phys. Rev. A 79, 063608.

Madsen, P. A. \& Hansen, A. B. 2012 Transient waves generated by a moving bottom obstacle: a new near-field solution. J. Fluid Mechanics 169, 429-464,.

MARchant, T. R. 2008 Undular bores and the initial-boundary value problem for the modified Korteweg - de Vries equation. Wave Motion 45, 540555.

Marchant, T. R. \& Smyth, N. F. 1990 The extended Korteweg-de Vries equation and the resonant flow of a fluid over topography. J. Fluid Mechanics 221, 263-287.

Marchant, T. R. \& Sмyтh, N. F. 2002 The initial boundary problem for the Korteweg - de Vries equation on the negative quarter-plane. J. Fluid Mechanics 458 (2020), 857-871.

Melville, W. K. \& Helfrich, K. R. 1987 Transcritical two-layer flow over topography. J. Fluid Mechanics 178, 31-52.

Michallet, H. \& BARThÉlemy, E. 1998 Experimental study of interfacial solitary waves. J. Fluid Mechanics 366, 159-177.

Smyth, N. 1987 Modulation theory solution for resonant flow over topography. Proc. Roy. Soc. Lond. A. 409, 79-97.

Trefethen, L. N. 2000 Spectral Methods in MATLAB. Society for Industrial and Applied Mathematics, Philadelphia.

Wan, W., Muenzel, S. \& Fleischer, J. W. 2010 Wave tunneling and hysteresis in nonlinear junctions. Phys. Rev. Lett. 104, 073903.

White, B. L. \& Helfrich, K. R. 2012 A general description of a gravity current front propagating in a two-layer stratified fluid. J. Fluid Mechanics 711, 545-575.

Whitham, G. B. 1965 Non-linear dispersive waves. Proc. Roy. Soc. A 283, 238-261.

Whitham, G. B. 1974 Linear and Nonlinear Waves. Wiley-Interscience, New York. 